\title{
Exploration of Aspherical Ejecta Properties in Type Ia Supernovae: Progenitor Dependence and Applications to Progenitor Classification
}

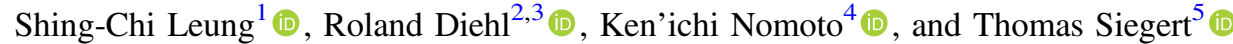 \\ ${ }^{1}$ TAPIR, Walter Burke Institute for Theoretical Physics, Mailcode 350-17, Caltech, Pasadena, CA 91125, USA; scleung@caltech.edu \\ ${ }_{2}^{2}$ Max-Planck-Institut für extraterrestrische Physik, Giessenbachstr. 1, D-85741 Garching, Germany \\ ${ }^{3}$ Excellence Cluster Universe, Technische Universität München, Boltzmannstr. 2, D-85748, Garching, Germany \\ ${ }^{4}$ Kavli Institute for the Physics and Mathematics of the Universe (WPI), The University of Tokyo Institutes for Advanced Study, The University of Tokyo, Kashiwa, \\ Chiba 277-8583, Japan \\ ${ }^{5}$ Center for Astrophysics and Space Sciences, University of California, San Diego, 9500 Gilman Drive, La Jolla, CA 92093, USA \\ Received 2019 September 23; revised 2020 October 28; accepted 2020 November 10; published 2021 March 12
}

\begin{abstract}
Several explosions of type Ia supernovae (SNe Ia) have been found to exhibit deviations from spherical symmetry upon closer inspection. Examples are the gamma-ray lines from SN 2014J as measured by INTEGRAL/SPI and morphology information from radioactive isotopes in older remnants such as Tycho. A systematic study of the effects of parameters such as ignition geometry and burning morphology in SNe Ia is still missing. We use a twodimensional hydrodynamics code with postprocessing nucleosynthesis and simulate the double detonations in a sub-Chandrasekhar-mass carbon-oxygen white dwarf starting from the nuclear runaway in the accumulated $\mathrm{He}$ envelope toward disruption of the white dwarf. We explore potential variety through four triggering scenarios that sample main asymmetry drivers. We further investigate their global effects on the aspherical structure of the ejecta based on individual elements. We apply the results to the well-observed SN 2014J and other recently observed SN remnants in order to illustrate how these new observational data, together with other observed quantities, help to constrain the explosion and progenitors of SNe Ia.
\end{abstract}

Unified Astronomy Thesaurus concepts: Type Ia supernovae (1728); Hydrodynamical simulations (767); Gammarays (637); Chemical abundances (224); Explosive nucleosynthesis (503); Supernova remnants (1667)

\section{Introduction}

\subsection{Observational Constraints on Type Ia Supernovae Progenitors}

Type Ia supernovae ( $\mathrm{SNe}$ Ia) have demonstrated a wide diversity (e.g., Taubenberger 2017). To trace the progenitor and the explosion mechanisms, supernova ( $\mathrm{SN}$ ) light curves and spectra are indispensable. (See, e.g., Hillebrand \& Niemeyer 2000; Maoz et al. 2014; Nomoto \& Leung 2018; and the Appendix for a summary of the progenitors and the explosion mechanism.) Explosion features can be extracted from the light-curve shape (e.g., Blondin et al. 2018), spectral lines such as Ca II and Ni II (e.g., Wilk et al. 2018), and the polarization (e.g., Bulla et al. 2016). The element abundance ratio can be the discriminant of current explosion models (e.g., Seitenzahl et al. 2009) by indicating the isotopic abundance ratios (e.g., ${ }^{57} \mathrm{Ni} /{ }^{56} \mathrm{Ni},{ }^{55} \mathrm{Fe} /{ }^{56} \mathrm{Ni}$, and ${ }^{44} \mathrm{Ti} /{ }^{56} \mathrm{Fe}$ ) and element abundance ratios (e.g., $\mathrm{Mn} / \mathrm{Fe}$ and $\mathrm{Ni} / \mathrm{Fe}$; see Mori et al. 2018). These techniques had been applied to a few well-observed SNe Ia, including SNe 2011fe, 2012cg, 2014J, and 2015F and SN remnant (SNR) 3C 397 (see recent work in, for example, Yamaguchi et al. 2015; Dave et al. 2017; Leung \& Nomoto 2017a, 2018; Shen et al. 2018; Zhou et al. 2021). The progenitor mass and metallicity are constrained by this method, which allows further identification of isotopic mass fractions of radioactive Ni isotopes in SN 2012cg (Graur et al. 2016) and SN 2014J (Yang et al. 2018) and stable Ni by the nebular IR spectra (see Dhawan et al. 2018, for the application to SN 2014J).

Radioactive isotopes with a longer half-life, e.g., ${ }^{44} \mathrm{Ti}$ with a half-year of $59 \mathrm{yr}$, can help to distinguish the different subclasses of $\mathrm{SNe}$ and their explosion mechanisms by their effects on the decline rate of the late-time light curve and measuring the absolute abundances (see, e.g., Fry et al. 2015).
Based on the tomography and morphology of SNRs, the progenitor constraints are cast on the SNR Tycho (Wang \& Li 2014; Lopez et al. 2015). The $\mathrm{S}$ and $\mathrm{Fe}$ spectral lines obtained from SNRs 0519-69.0, 0509-67.5, and N103B are further examined in the shock-heated nebulae (Seitenzahl et al. 2019). The heated matter can emit X-ray lines that provide direct constraints on the explosion mechanism, in particular the asphericity of SNe Ia.

Analysis of SNRs on larger scales, including the Small and Large Magellanic Clouds, can reveal the SN explosion history (Maggi et al. 2016, 2019). The study of particular elements, e.g., $\mathrm{Mn}$, can point out the major explosion mechanism (McWilliam et al. 2018; de los Reyes et al. 2020; Kobayashi et al. 2020).

The morphology reveals the inherent explosion asymmetry. For individual SNRs, the possible explosion progenitors can be inferred from their X-ray emission, which reveals the chemical abundances (Badenes et al. 2005, 2006, 2008). Differences in the interaction between the $\mathrm{SN}$ ejecta and the ambient medium can also point out the progenitor (Martínez-Rodríguez et al. 2018). The history of ejecta interaction with circumstellar matter (CSM) is contained in the late-time light curve as well. ${ }^{6}$

\subsection{Our Previous Studies of SNe Ia and Present Work on Asymmetries}

We have performed nucleosynthesis surveys of SN Ia explosions by the near-Chandrasekhar-mass white dwarf (WD) model using the turbulent deflagration model with the deflagration-detonation transition in Leung \& Nomoto (2018,

\footnotetext{
6 We refer interested readers to the related presentations in the conference "Progenitors of SNe Ia," available in http://bps.ynao.cas.cn/xzzx/201908/ t20190820_510006.html.
} 
hereafter Paper I), the sub-Chandrasekhar-mass WD model using the double-detonation model in Leung \& Nomoto (2020a, hereafter Paper II), and the near-Chandrasekhar-mass model for SNe Iax using the pure turbulent deflagration model in Leung \& Nomoto (2020b, hereafter Paper III). In these papers, we conducted an extended parameter survey of the SN Ia models aimed at understanding the implication of its observed diversity to progenitor model diversity, as well as constraints on explosion physics.

In Paper I, we considered models with the masses of $M=1.30-1.38 M_{\odot}$ and metallicity of $Z=0-5 Z_{\odot}$. The supersolar metallicity is suggested to explain the observed SNe Ia, including the SNR 3C $397\left(Z \sim 5 Z_{\odot}\right)$ and SN 2012cg $\left(Z \sim 3-5 Z_{\odot}\right)$. In Paper II, we considered WD models with $M=0.9-1.3 M_{\odot}, Z=$ $0-5 Z_{\odot}$, and He-envelope mass of $M_{\mathrm{He}}=0.05-0.2 M_{\odot}$. We show that sub-Chandrasekhar-mass models can also explain the isotopic ratio of nearby $\mathrm{SNe} \mathrm{Ia}$, as the near-Chandrasekhar-mass model does, but its Mn production cannot explain the nearby [Mn/Fe] trend taken from stars in the solar neighborhood. Explosions of WD models with $M \sim 1.2 M_{\odot}$ can provide the key to distinguish the two explosion channels. In Paper III, we specifically model SNe Iax with the pure turbulent deflagration model of the WDs with $M=1.30-1.39 M_{\odot}$ and $Z=Z_{\odot}$ (Leung \& Nomoto 2020b).

In the present Paper IV, we study the asymmetry of the WD models as suggested from SN 2014J, the SNR Tycho, and other features mentioned above. The ejecta geometry is primarily dependent on the explosion progenitor. The near-Chandrasekhar-mass model exploding by the turbulent deflagration model with the deflagration-detonation transition tends to explode spherically (see, e.g., Röpke et al. 2007, for recent threedimensional realizations). On the contrary, the sub-Chandrasekhar-mass model that explodes by the He-induced double detonations can generate large-scale asymmetry because of the off-center trigger of the explosion (see, e.g., Moll \& Woosley 2013; García-Senz et al. 2018; Gronow et al. 2020, for some recent three-dimensional realizations showing aspherical structures).

We thus focus on the sub-Chandrasekhar-mass models, which tend to have a more aspherical structure than the nearChandrasekhar-mass models. We note that in the literature, there is no extensive work examining how sub-Chandrasekharmass models exhibit a large-scale asphericity. In this Paper IV, we study for the first time how the different detonation mechanisms of the sub-Chandrasekhar-mass model can generate the ejecta deviated from the canonical spherical model by multidimensional simulations.

Specifically, we will clarify how the detonation triggered in the $\mathrm{He}$ envelope affects the ${ }^{56} \mathrm{Ni}$ distribution and ejecta structure in both position and velocity spaces. We also try to understand the underlying principles of the observed irregularities in SNRs.

We choose two-dimensional models so that we may test a larger number of models than three-dimensional models to uncover the global trend of the parameter landscape. Also, quasi-spherical SN 2014J (see Section 4) has encouraged us to explore models with a certain level of symmetry (e.g., rotation and reflection symmetry) assumed in two-dimensional models, instead of arbitrary models without explicit symmetry, as in three-dimensional models.

Ideally, three-dimensional models are naturally desired to match all features self-consistently. However, they are much more computationally expensive. As an exploratory study, we aim at searching the key properties in the detonation setting for the models to contain different features that might ultimately imprint in observational data. We want to understand what kind of shock interaction is necessary for generating the observed features, from which we may obtain hints of the initial detonation pattern. This will guide future three-dimensional simulations in setting up accurate initial models aiming to explain SN 2014J or other SNe.

\subsection{Paper Structure}

In Section 2, we describe our methodology and the models to be presented in this article. Then, we briefly review how we compute the explosion models of the sub-Chandrasekhar-mass $\mathrm{WD}$ as the progenitor, and we present the stellar parameters, explosion energetics, and essential nucleosynthetic products.

In Section 3, we first present how the near- and subChandrasekhar-mass WDs differ by their large-scale asymmetry. Then, we examine in detail how the explosion ejecta and its chemical composition depend on the viewing (ejecta) angle. We also predict the expected morphology by extracting the representative elements.

In Section 4, we present a detailed case study for SN 2014J to show how the asymmetry and other nucleosynthetic yields can be used for constraining the explosion mechanism and the progenitors of SNe Ia. We cast constraints on the progenitor mass, initial explosion geometry, He-envelope mass, and metallicity.

In Section 5, we further apply our results to some recently observed SNRs reported in the literature to demonstrate how the geometry can provide us the hints.

In Section 6, we discuss how this work is related to other hydrodynamics simulations in the literature.

\section{Methods and Models}

We use our two-dimensional hydrodynamics code developed for modeling the explosion models in this work. The code is based on high-order shock-capturing and time-discretization schemes, coupled with subgrid-scale turbulence models, flametracking schemes, and nuclear reaction networks of arbitrary sizes. We refer the interested reader to the instrumentation paper, which describes the prototype (Leung et al. 2015a). We have further extended the code to accommodate the physics in different explosion scenarios. Different extensions are described in detail for

(1) SNe Ia in Leung \& Nomoto (2017a, 2018, 2020a);

(2) electron-capture SNe in Leung \& Nomoto (2017b, 2019), and Zha et al. (2019b); Leung et al. (2020), and

(3) dark matter admixed compact objects in Leung et al. (2015b, 2019) and Zha et al. (2019a).

\subsection{Input Physics}

We follow Leung \& Nomoto (2020a) for SNe Ia using the sub-Chandrasekhar-mass WD models. We solve the twodimensional Euler equations in cylindrical coordinates. We further assume reflection symmetry of the $x-y$ plane so that we model only one quadrant of the star. We use a realistic Helmholtz equation of state (Timmes 1999) for describing the matter with free electrons with an arbitrary relativistic level and degeneracy, nuclei as a classical ideal gas, photons with Planck distribution, and electron-positron pair effects. Different from the near-Chandrasekhar-mass WD models, we include 
(1) the notation of He detonation in the simulation,

(2) its energy-generation prescription and timescale, and

(3) its propagation velocity.

We use the same solver for matter in nuclear statistical equilibrium (NSE), as it is independent of the original composition of the matter and only depends on the final density, temperature, and electron fraction. Level-set methods are used for tracing the contour of the He- and C-detonation fronts. In Paper II, we further performed a set of tests to justify the functionality of the code in the Appendix. They include that

(1) the C-detonation trigger is independent of the symmetry boundary we used, ${ }^{7}$

(2) the explosion energetics are insensitive to the resolution used,

(3) the shock convergence is less sensitive to the resolution, and

(4) the threshold of the detonation trigger is independent of the resolution.

We further showed in Paper II that our two-dimensional models give results that agree with contemporary one-, two-, and three-dimensional models found in the literature.

\subsection{Postprocess Nucleosynthesis}

In order to keep track of the detailed nucleosynthesis for constructing the isotope distribution, we use the tracer particle scheme (Travaglio et al. 2004; Seitenzahl et al. 2009; Townsley et al. 2016). It makes use of massless Lagrangian tracers. They follow the fluid motion and record their own thermodynamical trajectories. The particles are "massless" in that they do not change the fluid motion. After the hydrodynamical simulations, the tracers are postprocessed with a large nuclear reaction network (a 495-isotope network containing isotopes from ${ }^{1} \mathrm{H}$ to ${ }^{91} \mathrm{Tc}$; Timmes et al. 2000). After postprocessing, the spatial distributions of specific isotopes, such as ${ }^{16} \mathrm{O},{ }^{28} \mathrm{Si}$, and ${ }^{56} \mathrm{Ni}$, are extracted.

\subsection{Models}

In this work, based on the formalism of our previous works, we examine further SN Ia models in the range between 0.95 and $1.0 M_{\odot}$, and we extend systematically to different initial He-detonation structures. We evolve WDs from the onset of $\mathrm{He}$ detonation until no significant exothermic reactions take place. This can be because the WD is disrupted completely by both types of detonation, or the $\mathrm{He}$ detonation fails to trigger the second detonation and quenches. In Table 1, we list the initial densities and temperatures used in our models. As indicated by observational data, we consider the He-envelope mass $M_{\mathrm{He}}$ from 0.05 to $0.10 M_{\odot}$.

In the table, we also list other related parameters, including the central density $\rho_{c}$ and interface density $\rho_{\mathrm{He}}$. Among all of our considered models, the densities range from $\sim 10^{7}$ to $10^{8} \mathrm{~g} \mathrm{~cm}^{-3}$, while the $\mathrm{He}$ interface is from $\sim 10^{5}$ to $10^{6} \mathrm{~g} \mathrm{~cm}^{-3}$. Notice that this allows a major part of the star

\footnotetext{
We explored whether modeling the two-bubble structure using a hemisphere and quadrant gives rise to different results. We showed that indeed, the detonation waves collide identically, as if they are laminar waves at the imposed reflective boundary. In fact, this scenario provides a more stringent test of how robustly the second detonation can be triggered because there is no geometric convergence taking place near the reflective boundary. As a result, the required He-envelope mass predicted by this assumption is the upper limit. This value can be drastically reduced as geometric effects become stronger.
}

to carry out complete burning from CO matter to ashes in NSE at a density $>5 \times 10^{7} \mathrm{~g} \mathrm{~cm}^{-3}$.

In general, three types of events can result. In the table, N/A means that no $\mathrm{C}$ detonation occurs; this happens when the $\mathrm{He}$ detonation is too weak (without the possible geometric convergence) to heat the $\mathrm{CO}$ matter to a sufficient temperature, or collision such that the shock propagates into the core. The results "Cen." and "Off" stand for centered and off-center detonation, respectively. We also list the yielded ${ }^{56} \mathrm{Ni}$ and ${ }^{57} \mathrm{Ni}$ masses, obtained at the end of the simulations, where most exothermic reactions have ceased.

In Figure 1, we depict the four scenarios used in this work. They include a bubble (B type), a ring ( $\mathrm{R}$ type), a bubble and a ring (D type), and a sphere (S type). This spans the possible initial He runaway from the lowest to the highest symmetry. In the figure, the cross sections of the WD progenitor are drawn. The B-type detonation corresponds to two bubbles, one at the north pole and one at the south pole.

In order to realize the one-bubble event, simulations explicitly modeling the hemisphere are necessary. We recall that the $\mathrm{C}$ detonation that is triggered is very similar to the $\mathrm{R}$ type, since, in this configuration, the $\mathrm{C}$ detonation always starts after the shock converges at the opposite "pole" from where the detonation is initialized. Therefore, we may refer to R-type series to trace how the detonation takes place. We note that, computationally, the detonation trigger is identical, as indicated by Appendix B in Paper II.

\section{Representation of Asphericity of SNe Ia}

In this section, we examine how the asphericity of an $\mathrm{SN}$ Ia can be embodied by its observables. We first compare the typical ejecta structure from our two-dimensional simulations for the near- and sub-Chandrasekhar models to see how the progenitor mass affects the asphericity. Then we focus on the sub-Chandrasekhar-mass model to show how the initial detonation affects the ejecta distribution, velocity, and remnant morphology.

\subsection{Near-Chandrasekhar-mass WD versus Sub- Chandrasekhar-mass WD}

In this work, we focus on the asymmetry of the subChandrasekhar-mass WD. We do not focus on the nearChandrasekhar-mass WD because we observe that typical SN Ia models from the near-Chandrasekhar-mass WD tend to have a more spherical structure. Here we present a comparison to outline the large-scale asymmetry in both models. For the details of these models, we refer interested readers to Papers I, II, and III for more detailed descriptions and implementation.

The two models are chosen from our benchmark models. Both models are chosen to represent the "normal" SN Ia by its ${ }^{56} \mathrm{Ni}$ production, $\sim 0.6 M_{\odot}$. Furthermore, the near-Chandrasekhar-mass model also produces the necessary amount of ${ }^{55} \mathrm{Mn}$ for reproducing the trends of stellar abundance near the solar neighborhood.

In the top panel of Figure 2, we show the temperature in a section through the star of the near-Chandrasekhar-mass model at $\sim 4 \mathrm{~s}$ after the explosion. The model assumes turbulent deflagration with a deflagration-detonation transition. The initial nuclear runaway assumes a centered deflagration wave with angular perturbations. The near-Chandrasekhar-mass WD 
Table 1

Initial Conditions, Explosion Energetics, and Global Nucleosynthetic Results of the Sub-Chandrasekhar-mass SN Ia Models Presented in This Work

\begin{tabular}{|c|c|c|c|c|c|c|c|c|c|c|c|c|}
\hline Model & $M$ & $M_{\mathrm{He}}$ & $M_{\mathrm{CO}}$ & Flame & $R$ & $R_{\mathrm{He}}$ & $\rho_{c}$ & $\rho_{\mathrm{He}}$ & 2nd Runaway? & Type? & $M\left({ }^{56} \mathrm{Ni}\right)$ & $M\left({ }^{57} \mathrm{Ni}\right)$ \\
\hline 095-050-B & 0.95 & 0.05 & 0.90 & B & 6710 & 4760 & 2.23 & 0.06 & No & N/A & $\mathrm{N} / \mathrm{A}$ & N/A \\
\hline 095-050-R & 0.95 & 0.05 & 0.90 & $\mathrm{R}$ & 6710 & 4760 & 2.23 & 0.06 & Yes & Off & 0.11 & $3.04 \times 10^{-3}$ \\
\hline 095-050-D & 0.95 & 0.05 & 0.90 & D & 6710 & 4760 & 2.23 & 0.06 & No & $\mathrm{N} / \mathrm{A}$ & $\mathrm{N} / \mathrm{A}$ & $\mathrm{N} / \mathrm{A}$ \\
\hline $095-050-\mathrm{S}$ & 0.95 & 0.05 & 0.90 & $\mathrm{~S}$ & 6710 & 4760 & 2.23 & 0.06 & Yes & Cen. & 0.45 & $1.14 \times 10^{-2}$ \\
\hline 095-100-B & 0.95 & 0.10 & 0.85 & B & 6710 & 4330 & 2.23 & 0.12 & No & $\mathrm{N} / \mathrm{A}$ & $\mathrm{N} / \mathrm{A}$ & $\mathrm{N} / \mathrm{A}$ \\
\hline 095-100-R & 0.95 & 0.10 & 0.85 & $\mathrm{R}$ & 6710 & 4330 & 2.23 & 0.12 & Yes & Off & 0.31 & $8.65 \times 10^{-3}$ \\
\hline 095-100-D & 0.95 & 0.10 & 0.85 & $\mathrm{D}$ & 6710 & 4330 & 2.23 & 0.12 & Yes & Off & 0.29 & $8.63 \times 10^{-3}$ \\
\hline 095-100-S & 0.95 & 0.10 & 0.85 & $S$ & 6710 & 4330 & 2.23 & 0.12 & Yes & Cen. & 0.48 & $1.28 \times 10^{-2}$ \\
\hline 100-050-B & 1.00 & 0.05 & 0.95 & B & 6180 & 4350 & 3.21 & 0.09 & No & $\mathrm{N} / \mathrm{A}$ & $\mathrm{N} / \mathrm{A}$ & N/A \\
\hline $100-050-\mathrm{R}$ & 1.00 & 0.05 & 0.95 & $\mathrm{R}$ & 6180 & 4350 & 3.21 & 0.09 & Yes & Off & 0.31 & $8.16 \times 10^{-3}$ \\
\hline 100-050-D & 1.00 & 0.05 & 0.95 & $\mathrm{D}$ & 6180 & 4350 & 3.21 & 0.09 & Yes & Off & 0.08 & $2.31 \times 10^{-3}$ \\
\hline $100-050-S$ & 1.00 & 0.05 & 0.95 & $\mathrm{~S}$ & 6180 & 4350 & 3.21 & 0.09 & Yes & Cen. & 0.60 & $1.60 \times 10^{-2}$ \\
\hline 100-100-B & 1.00 & 0.10 & 0.90 & B & 6180 & 3980 & 3.21 & 0.16 & Yes & Off & 0.35 & $1.14 \times 10^{-2}$ \\
\hline $100-100-R$ & 1.00 & 0.10 & 0.90 & $\mathrm{R}$ & 6180 & 3980 & 3.21 & 0.16 & Yes & Off & 0.46 & $1.30 \times 10^{-2}$ \\
\hline 100-100-D & 1.00 & 0.10 & 0.90 & $\mathrm{D}$ & 6180 & 3980 & 3.21 & 0.16 & Yes & Off & 0.44 & $1.26 \times 10^{-2}$ \\
\hline $100-100-S$ & 1.00 & 0.10 & 0.90 & $\mathrm{~S}$ & 6180 & 3980 & 3.21 & 0.16 & Yes & Cen. & 0.62 & $1.74 \times 10^{-2}$ \\
\hline 105-050-B & 1.05 & 0.05 & 1.00 & B & 5300 & 4110 & 4.33 & 0.10 & No & $\mathrm{N} / \mathrm{A}$ & $\mathrm{N} / \mathrm{A}$ & $\mathrm{N} / \mathrm{A}$ \\
\hline 105-050-R & 1.05 & 0.05 & 1.00 & $\mathrm{R}$ & 5300 & 4110 & 4.33 & 0.10 & Yes & Off & 0.48 & $1.24 \times 10^{-2}$ \\
\hline 105-050-D & 1.05 & 0.05 & 1.00 & $\mathrm{D}$ & 5300 & 4110 & 4.33 & 0.10 & Yes & Off & 0.48 & $1.37 \times 10^{-2}$ \\
\hline $105-050-\mathrm{S}$ & 1.05 & 0.05 & 1.00 & $\mathrm{~S}$ & 5300 & 4110 & 4.33 & 0.10 & Yes & Cen. & 0.76 & $1.63 \times 10^{-2}$ \\
\hline 105-100-B & 1.05 & 0.10 & 0.95 & B & 5300 & 3730 & 4.33 & 0.19 & Yes & Off & 0.49 & $1.65 \times 10^{-2}$ \\
\hline 105-100-R & 1.05 & 0.10 & 0.95 & $\mathrm{R}$ & 5300 & 3730 & 4.33 & 0.19 & Yes & Off & 0.59 & $1.78 \times 10^{-2}$ \\
\hline 105-100-D & 1.05 & 0.10 & 0.95 & $\mathrm{D}$ & 5300 & 3730 & 4.33 & 0.19 & Yes & Off & 0.59 & $1.80 \times 10^{-2}$ \\
\hline $105-100-S$ & 1.05 & 0.10 & 0.95 & $S$ & 5300 & 3730 & 4.33 & 0.19 & Yes & Cen. & 0.70 & $2 \times 10^{-2}$ \\
\hline 110-050-B & 1.10 & 0.05 & 1.05 & B & 4930 & 3800 & 6.17 & 0.13 & No & $\mathrm{N} / \mathrm{A}$ & $\mathrm{N} / \mathrm{A}$ & N/A \\
\hline $110-050-R$ & 1.10 & 0.05 & 1.05 & $\mathrm{R}$ & 4930 & 3800 & 6.17 & 0.13 & Yes & Off & 0.68 & $1.90 \times 10^{-2}$ \\
\hline 110-050-D & 1.10 & 0.05 & 1.05 & $\mathrm{D}$ & 4930 & 3800 & 6.17 & 0.13 & Yes & Off & 0.60 & $1.72 \times 10^{-2}$ \\
\hline $110-050-\mathrm{S}$ & 1.10 & 0.05 & 1.05 & S & 4930 & 3460 & 6.17 & 0.13 & Yes & Cen. & 0.82 & $1.90 \times 10^{-2}$ \\
\hline 110-100-B & 1.10 & 0.10 & 1.00 & B & 4930 & 3460 & 6.17 & 0.24 & Yes & Off & 0.61 & $2.10 \times 10^{-2}$ \\
\hline $110-100-\mathrm{R}$ & 1.10 & 0.10 & 1.00 & $\mathrm{R}$ & 4930 & 3460 & 6.17 & 0.24 & Yes & Off & 0.75 & $2.37 \times 10^{-2}$ \\
\hline 110-100-D & 1.10 & 0.10 & 1.00 & $\mathrm{D}$ & 4930 & 3460 & 6.17 & 0.24 & Yes & Off & 0.70 & $2.21 \times 10^{-2}$ \\
\hline $110-100-S$ & 1.10 & 0.10 & 1.00 & $\mathrm{~S}$ & 4930 & 3460 & 6.17 & 0.24 & Yes & Cen. & 0.81 & $2.43 \times 10^{-2}$ \\
\hline \multicolumn{13}{|l|}{ Observations } \\
\hline Lower limit & 0.7 & 0.03 & & & & & & & & & 0.4 & $2.32 \times 10^{-2}$ \\
\hline Upper limit & 3.1 & 0.09 & & & & & & & & & 0.7 & $5.53 \times 10^{-2}$ \\
\hline
\end{tabular}

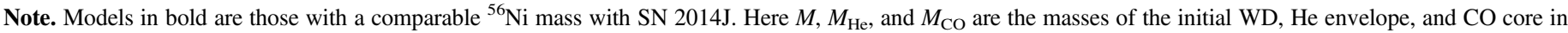

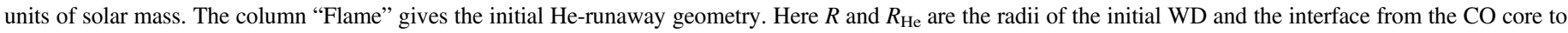

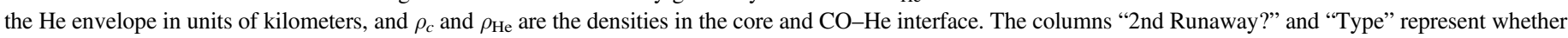

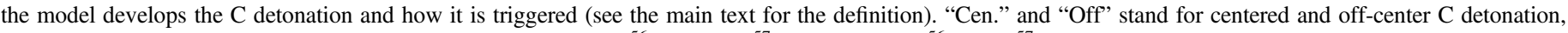

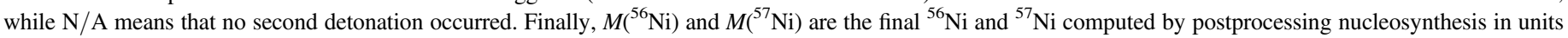
of $M_{\odot}$.

has a mass of $1.37 M_{\odot}$ and a central density of $3 \times 10^{9} \mathrm{~g} \mathrm{~cm}^{-3}$. A centered-flame $c 3$ (with three-finger structure) is used as the initial nuclear runaway. The finger structure is used to enhance hydrodynamical instabilities for the asphericity. The subChandrasekhar-mass model has a total mass of $1.1 M_{\odot}$ with a helium envelope mass of $0.1 M_{\odot}$ and a central density of $\sim 6 \times 10^{7} \mathrm{~g} \mathrm{~cm}^{-3}$. A one-bubble configuration is placed along the rotation axis.

For our model, at $t \sim 4-5 \mathrm{~s}$, the global distribution of ejecta structure begins to be frozen. Secondary features, including Rayleigh-Taylor instabilities ("mushrooms"), still continue to grow. But most features are smoothed by the expansion, leaving a surface close to spherical. We can observe that by the external detonation transition, the detonation wave always wraps around the aspherical ash produced during deflagration.
Surface asphericity in temperature appears to be significant in the Chandrasekhar-mass model at a radius of $\sim 30,000 \mathrm{~km}$. However, the matter at the surface is mostly $\mathrm{C}$ and $\mathrm{O}$. The area of interest, where iron-peak elements are synthesized, does not show much asphericity. They are produced primarily in the core, which means it is unlikely that large-scale asymmetry features from ${ }^{56} \mathrm{Ni}$ and ${ }^{56} \mathrm{Co}$ can be generated and exposed. In this work, the core corresponds to the CO-rich matter under the He envelope.

In the bottom panel of Figure 2, we show the temperature profile of a sub-Chandrasekhar-mass $\mathrm{CO}$ WD with an $\mathrm{He}$ envelope for comparison. The sub-Chandrasekhar-mass model shows a more explicit large-scale asphericity. The detonation is triggered near the "equator." As a result, the He detonation is stronger near the equator, as shown by the hot spot $60,000 \mathrm{~km}$ 


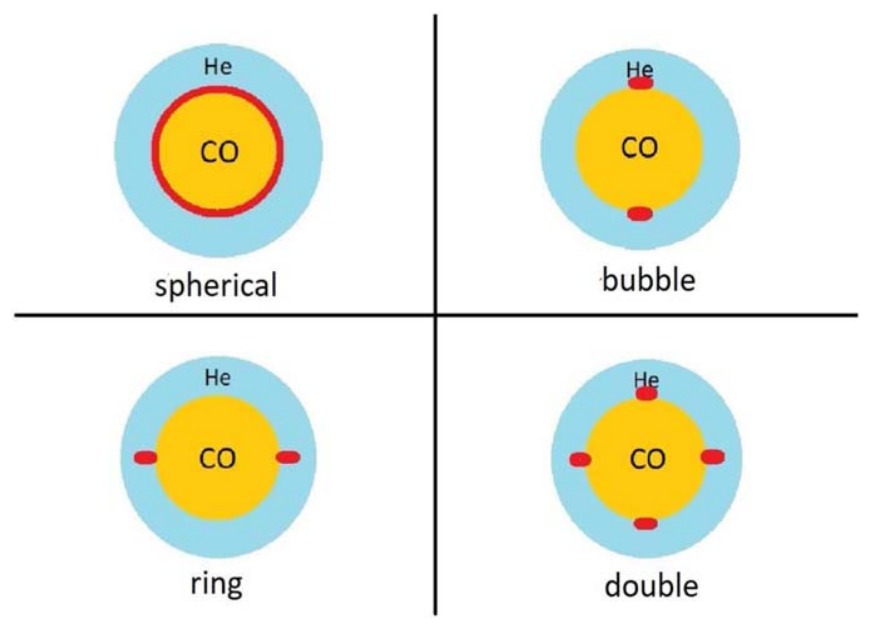

Figure 1. Graphical illustrations of the initial He-detonation configuration used in this paper. The figure depicts the cross sections of initial CO WDs with $\mathrm{He}$ envelopes and the initial He detonation put in by hand. The $\mathrm{S}$ (spherical), B (bubble), R (ring), and D (double) types of geometry are presented. The orange and light blue regions stand for the $\mathrm{CO}$ - and He-rich regions. The red region stands for the zone that is assumed to be burned already at the beginning of the simulations.

closer to the symmetry axis. Since the $\mathrm{C}$ detonation propagates from the place near the equator to the center and then outward, the inner ejecta from the $\mathrm{C}$ detonation has a preferred direction along the rotation axis. The high-velocity flow along this direction will be responsible for the later large-scale asphericity.

\subsection{Detonation Geometry-Induced Asphericity}

Having shown that the sub-Chandrasekhar-mass WD is capable of generating large-scale asymmetry, we further examine how the distribution of ${ }^{56} \mathrm{Ni}$ deviates from spherical symmetry. In Figure 3, we show the final distributions of the tracer particles, which are the mass fraction contours of ${ }^{16} \mathrm{O}$, ${ }^{28} \mathrm{Si}$, and ${ }^{56} \mathrm{Ni}$. As examples, we consider models $100-100-\mathrm{R}$, 100-100-B, and 100-100-S.

For model 100-100-S, we can see that all of the Ni-rich matter is covered by the thick envelope. We choose this series because all of these models produce comparable ${ }^{56} \mathrm{Ni}$ mass as a normal SN Ia.

The S-type model does not show any significant amount of ${ }^{56} \mathrm{Ni}$ near the surface. The distribution confirms that the initial spherical symmetry makes the detonation wave propagate only spherically. Except for small-scale perturbation coming from discretization effects from cylindrical to spherical coordinates, the large-scale distribution is, to a good approximation, spherical. The detonation waves do not collide with each other except at the stellar core when the detonation waves converge. There is no detonation wave interaction by shock collision or convergence in the $\mathrm{He}$ envelope. The amount of ${ }^{56} \mathrm{Ni}$ production becomes lower in the envelope. Hence, this model is less likely to explain the observed early ${ }^{56} \mathrm{Ni}$ decay line.

The R- and B-type models show more near-surface ${ }^{56} \mathrm{Ni}$. The ${ }^{56} \mathrm{Ni}$ is ejected more along the polar direction for the B-type detonation; meanwhile, the R-type detonation ejects matter more spherically but in a more elongated manner compared with S-type model. The D-type model also behaves similarly to the R-type model, except that the ${ }^{56} \mathrm{Ni}$ around the equator is lower in abundance.
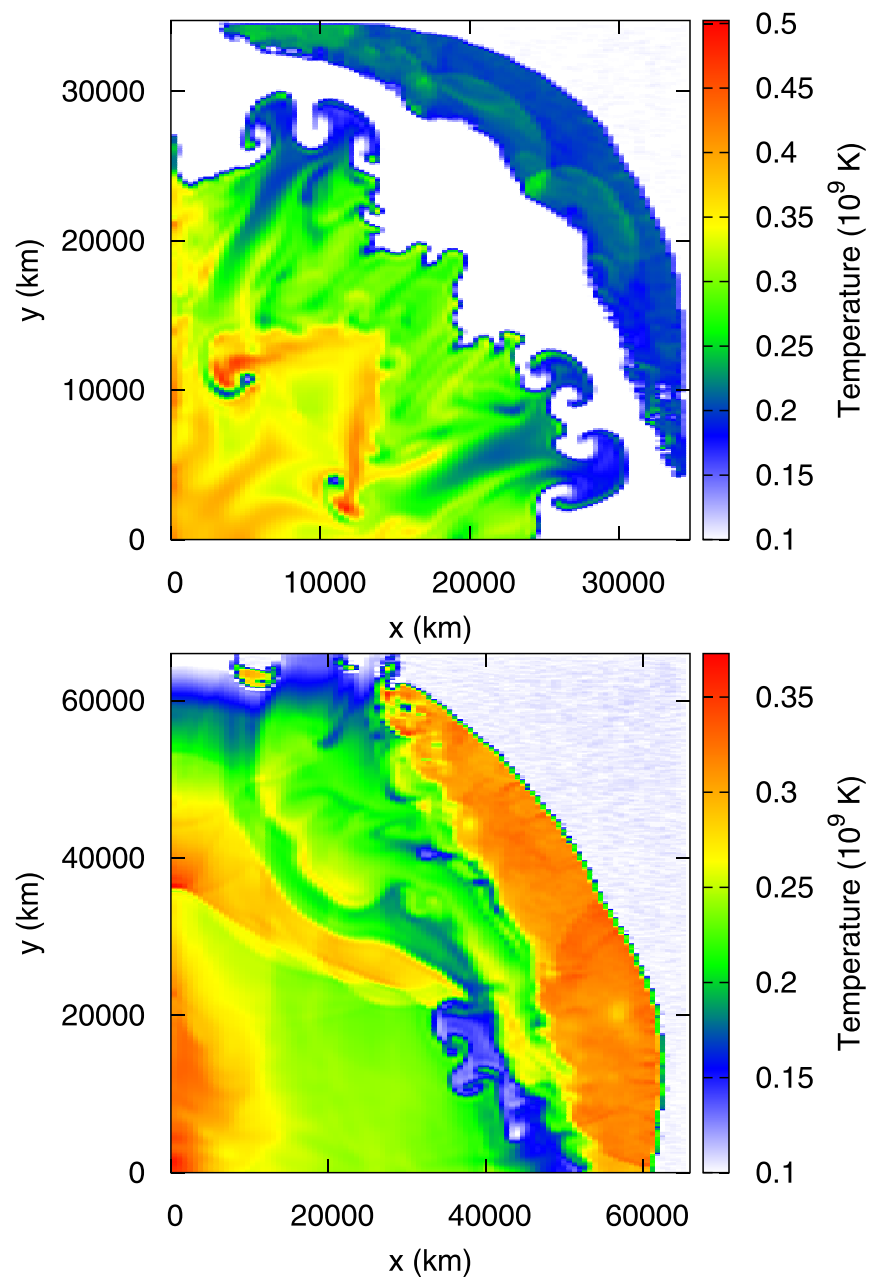

Figure 2. (Top panel) Color temperature profile of our benchmark SN Ia models from the explosion of a near-Chandrasekhar-mass WD at $\sim 4 \mathrm{~s}$ after the nuclear runaway has started (see Paper II for more details). (Bottom panel) Similar to the top panel but from a sub-Chandrasekhar-mass WD (this work).

We recall that it is the asynchronous burning of helium, coupled with the geometric convergence, that creates the observed asphericity. The asynchronous burning allows He with the same initial density to be burned at different times. When the detonation time is delayed, the density of the $\mathrm{He}$ matter decreases, thus making it less likely to generate ${ }^{56} \mathrm{Ni}$ in the first place. On the contrary, the geometric convergence creates the hot spot for robustly triggering ${ }^{56} \mathrm{Ni}$ synthesis. However, the effect of geometric convergence is more localized. In a similar way, the detonation wave collision can create the hightemperature and high-density zones for synthesizing ${ }^{56} \mathrm{Ni}$.

\subsection{Velocity Distribution of Ejecta}

One of the features in SN 2014J is the early-time ${ }^{56} \mathrm{Ni}$ signal and time-dependent velocity for late-time ${ }^{56} \mathrm{Co}$ (see Section 4). It is therefore interesting to further study how the initial detonation configuration can give rise to the diversity of the isotope velocity distributions. In Figure 4, we plot the ejecta distribution in the velocity space to analyze how the detonation affects the final ejecta distribution. The distribution is angular-averaged. More samples of SNe Ia show similar early premaximum bumps (see, e.g., Jiang et al. 2017, 2018). The possibility of these SNe Ia forming a subclass shows that $\mathrm{SNe}$ Ia with observable $\mathrm{He}$ burning features can have a common evolutionary path. 

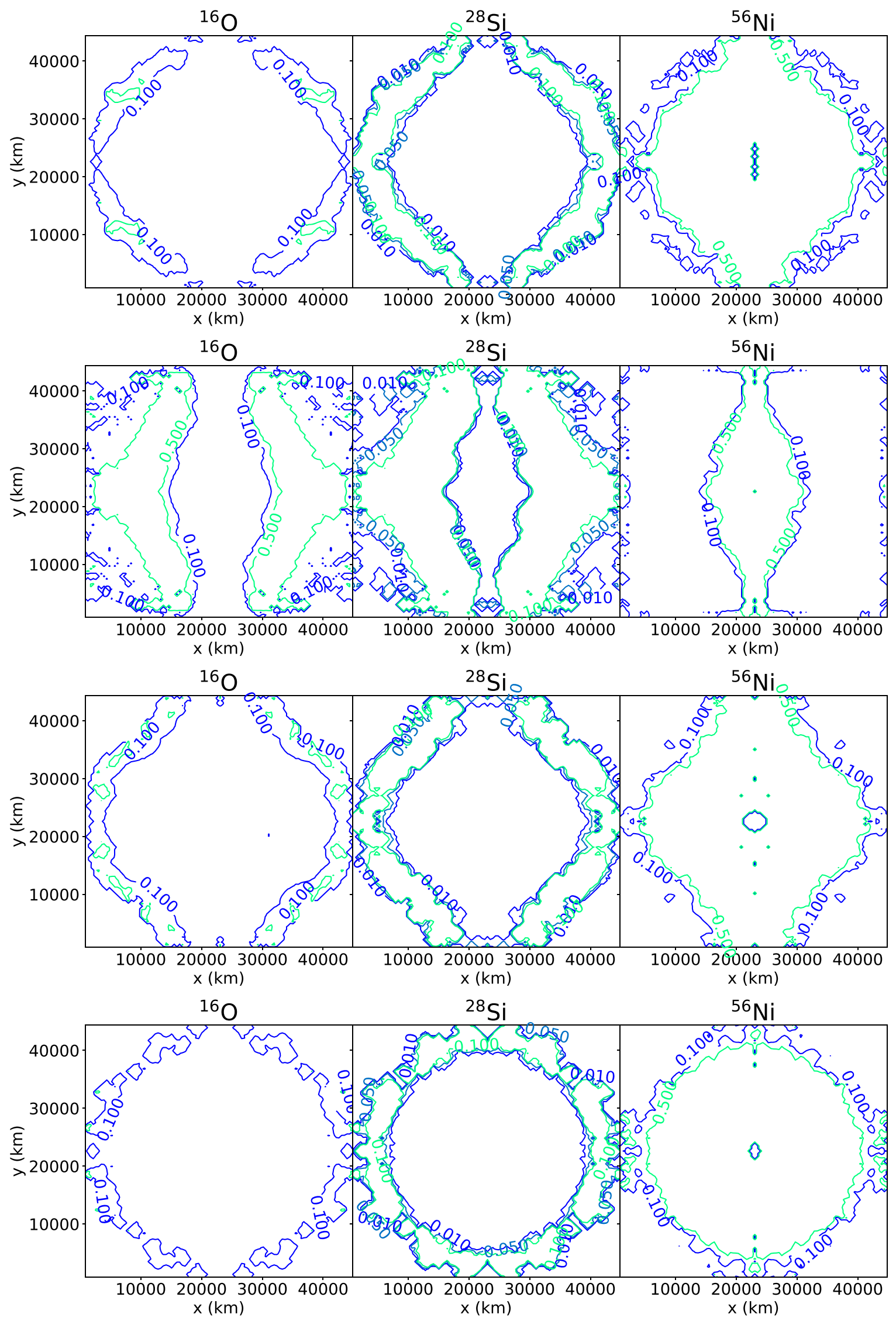

Figure 3. Mass fraction distributions of ${ }^{16} \mathrm{O},{ }^{28} \mathrm{Si}$, and ${ }^{56} \mathrm{Ni}$ for the explosion models $100-100-\mathrm{R}$ (first panel), 100-100-B (second panel), 100-100-D (third panel), and 100-100-S (fourth panel). The numbers stand for the corresponding contours of the mass fraction for the particular isotope. 

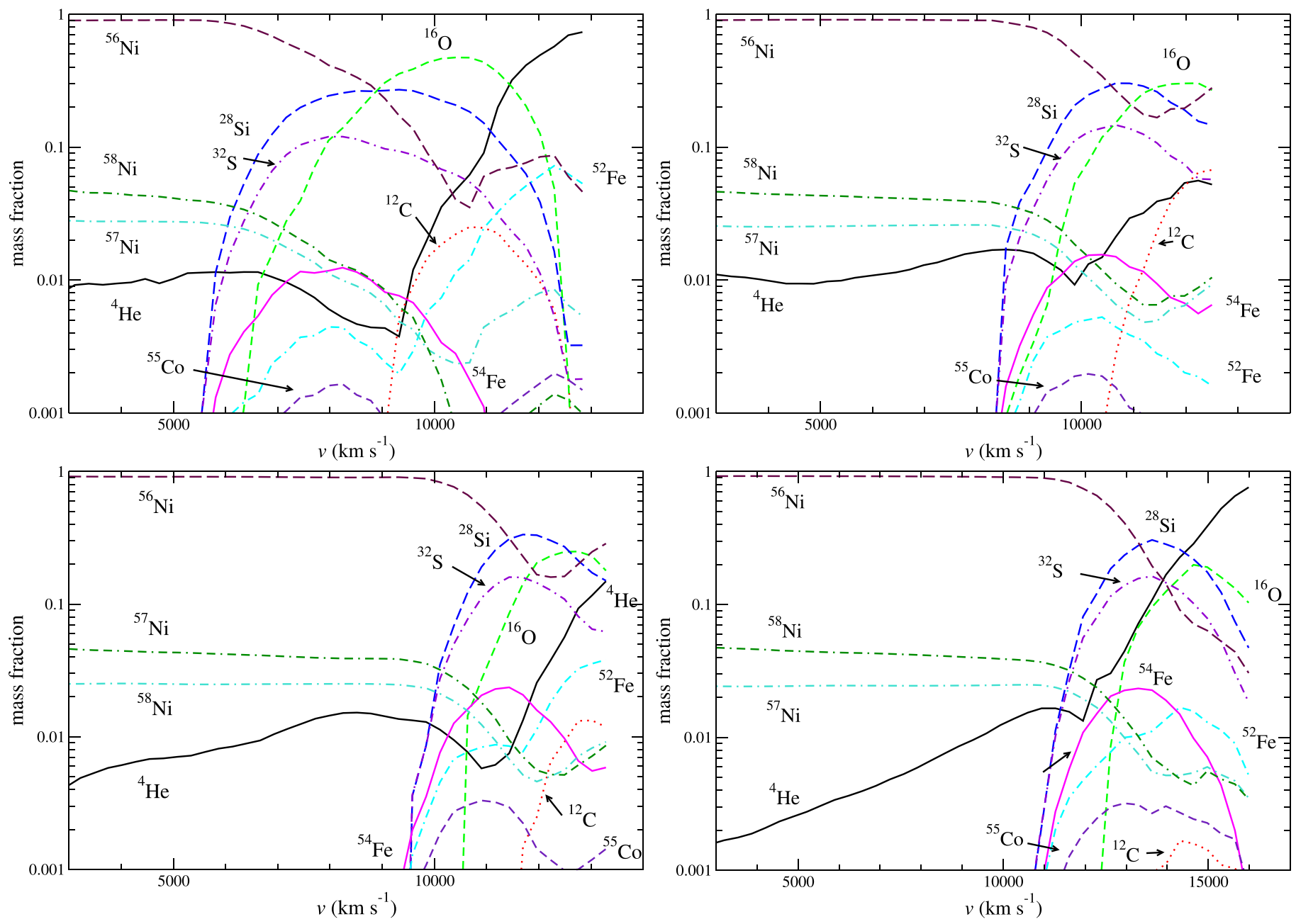

Figure 4. Velocity distributions of the ejecta for models 100-100-B (top left), 100-100-D (top right), 100-100-R (bottom left), and 100-100-S (bottom right).

Furthermore, such early gamma-ray flux can be another important sign for future telescopes to capture the early optical evolution of these SNe (Wang et al. 2019).

For model 100-100-B, the one-bubble configuration allows ejecta to be concentrated with ${ }^{56} \mathrm{Ni}$-rich material (see Figure 3) near the equator. As a result, there is a multilayered distribution of ${ }^{56} \mathrm{Ni}$. Below $6000 \mathrm{~km} \mathrm{~s}^{-1}$, the ejecta are filled with ${ }^{56-58} \mathrm{Ni}$. From 6000 to $8000 \mathrm{~km} \mathrm{~s}^{-1},{ }^{28} \mathrm{Si}$ and ${ }^{32} \mathrm{~S}$ are the major isotopes. For $8000-11,000 \mathrm{~km} \mathrm{~s}^{-1}$, unburned oxygen is the major element, and outside $\mathrm{He}$ is in the main element in the ejecta. We remark that the ${ }^{56} \mathrm{Ni}$ distribution is not monotonically decreasing, as compared to the classical spherical model. It first drops around $10,000 \mathrm{~km} \mathrm{~s}^{-1}$, showing that the detonation reaches the low-density region for $\mathrm{C}$ burning. After that, the mass fraction of ${ }^{56} \mathrm{Ni}$ rises again and quickly drops in its abundance. This shows that the shock collision in the $\mathrm{He}$ envelope allows formation of ${ }^{56} \mathrm{Ni}$ directly. But the shock is not strong enough to channel the outburst of ${ }^{56} \mathrm{Ni}$, as seen by the covering layer of ${ }^{4} \mathrm{He}$.

For model 100-100-D, the strong collision away from the axis allows an outburst of ${ }^{56} \mathrm{Ni}$ during ${ }^{4} \mathrm{He}$ burning at early time. This is also reflected in the ejecta distribution. Ejecta with a velocity below $10,000 \mathrm{~km} \mathrm{~s}^{-1}$ are again filled with $\mathrm{Ni}$ isotopes. Here ${ }^{28} \mathrm{Si}$ and ${ }^{32} \mathrm{~S}$ are the major isotopes in the velocity range $10,000-11,000 \mathrm{~km} \mathrm{~s}^{-1}$. Products of incomplete $\mathrm{C}$ burning, such as unburned ${ }^{16} \mathrm{O}$, can be found most abundantly up to
$12,000 \mathrm{~km} \mathrm{~s}^{-1}$. Outside that, ${ }^{56} \mathrm{Ni}$ and ${ }^{16} \mathrm{O}$ are the major isotopes. From this, it can be seen that multiple ignitions allow ${ }^{4} \mathrm{He}$ to be burned quickly along the $\alpha$-chain. Such outermost ${ }^{56} \mathrm{Ni}$ can be readily ejected and seen through its decay.

For model 100-100-R, the geometric convergence takes place at the pole that is strong enough to create similar pinching to the He envelope. The ejecta are covered with intermediate mass elements (IMEs) from 10,000 to $11,000 \mathrm{~km} \mathrm{~s}^{-1}$ and ${ }^{16} \mathrm{O}$ from 11,000 to $12,000 \mathrm{~km} \mathrm{~s}^{-1}$. The outermost layer is mixed with ${ }^{56} \mathrm{Ni}$ and ${ }^{4} \mathrm{He}$ with hints of ${ }^{28} \mathrm{Si}$ and ${ }^{32} \mathrm{~S}$. Similar to model 100-100-D, there is a thin layer of IMEs between 10,000 and $11,000 \mathrm{~km} \mathrm{~s}^{-1}$ and then incomplete C-burning products between 11,000 and $12,000 \mathrm{~km} \mathrm{~s}^{-1}$. The outermost ejecta are a mixture of ${ }^{56} \mathrm{Ni},{ }^{4} \mathrm{He},{ }^{28} \mathrm{Si},{ }^{32} \mathrm{~S}$, and ${ }^{16} \mathrm{O}$.

For model 100-100-S, the spherical detonation allows a stratified structure in the ejecta. Ejecta with a velocity below $12,000 \mathrm{~km} \mathrm{~s}^{-1}$ are dominated by ${ }^{56} \mathrm{Ni},{ }^{57} \mathrm{Ni}$, and ${ }^{58} \mathrm{Ni}$. The IMEs, including ${ }^{28} \mathrm{Si}$ and ${ }^{32} \mathrm{~S}$, are mostly found between 12,000 and $14,000 \mathrm{~km} \mathrm{~s}^{-1}$. Ejecta with a velocity $>14,000 \mathrm{~km} \mathrm{~s}^{-1}$ are occupied by ${ }^{4} \mathrm{He}$.

Comparing these four models demonstrates the possibility of mixing ${ }^{56} \mathrm{Ni}$ at high velocity, namely at the outermost ejecta for the D and B types. The oblique shock and geometric convergence of detonation inside the He envelope can provide the necessary thrust for channeling the ${ }^{56} \mathrm{Ni}$ produced in the $\mathrm{He}$ detonation to the outermost part of the ejecta. When the ejecta 

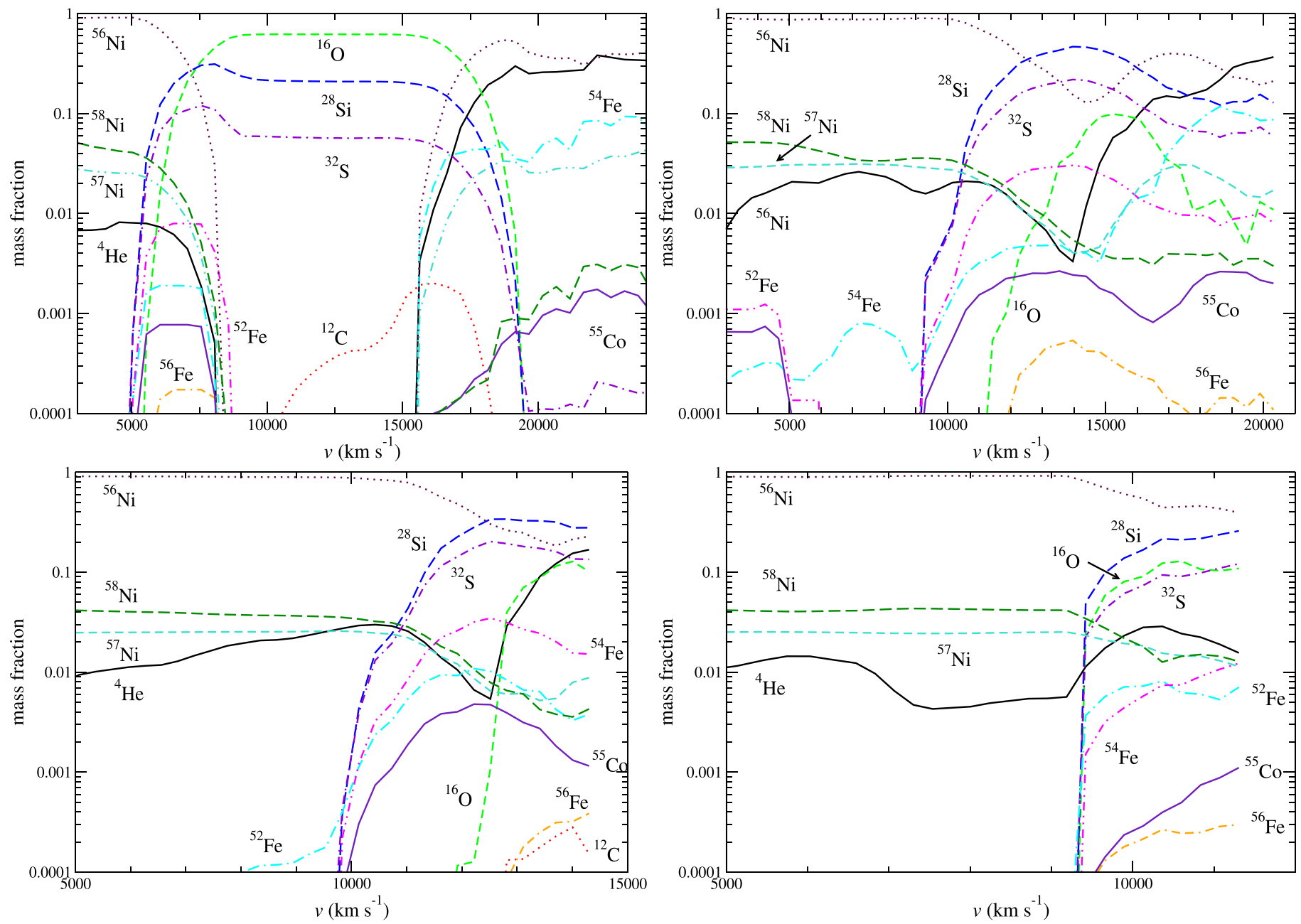

Figure 5. (Top left panel) Ejecta compositions in the velocity space for model 100-100-B along the angular slice from $0^{\circ}$ to $9^{\circ}$ from the rotation axis. (Top right panel) Same as the top left panel but for the angular slice $81^{\circ}-90^{\circ}$ from the rotation axis. (Bottom left panel) Ejecta compositions in the velocity space for model $100-100-\mathrm{R}$ along the angular slice from $0^{\circ}$ to $9^{\circ}$ from the rotation axis. (Bottom right panel) Same as the bottom left panel but for the angular slice $81^{\circ}-90^{\circ}$ from the rotation axis.

quickly expands, the gamma rays produced by the decay of ${ }^{56} \mathrm{Ni}$ into ${ }^{56} \mathrm{Co}$ may be directly seen after the surface matter becomes optically thin.

\subsection{Directional Dependence of Ejecta}

In the previous section, we examine the angular average ejecta velocity composition, and we show that the ejecta composition depends on the explosion geometry. Here we further analyze the ejecta composition by choosing specific angular slices. In particular, we choose the angular slices at $0^{\circ}-$ $9^{\circ}$ from the rotation axis and from the symmetry axis (i.e., $81^{\circ}-$ $90^{\circ}$ from the rotation axis) to contrast the ejecta composition. This demonstrates the difference between metal production with and without geometric convergence.

\subsubsection{Bubble-type Explosion}

The one-bubble configuration has been shown to be weaker than its one-ring counterpart due to the lack of shock convergence. The explosion is weaker with lower ${ }^{56} \mathrm{Ni}$ production. In the top left and right panels of Figure 5, we plot the ejecta distribution in the velocity space of model 100100-B for two angular slices near the rotation axis (left) and symmetry axis (right).
The two slices show very different ejecta structures. The ejecta along the rotation axis is slightly faster than the rotation axis. This is because the initial detonation triggered along the poles. However, we remark that this does not mean there is more substance along the axis because the velocity space does not directly follow the mass coordinate. The iron-peak elements in the core also differ a lot. Along the poles, the representatives of iron-peak elements, including ${ }^{56,57,58} \mathrm{Ni}$, occupy the innermost $\sim 7000 \mathrm{~km} \mathrm{~s}^{-1}$, and then the abundance quickly drops off. On the other hand, the iron-peak element-rich core extends up to $12,000 \mathrm{~km} \mathrm{~s}^{-1}$ along the equator. The large difference comes from the $\mathrm{C}$ detonation. It is triggered near the equator. As a result, the $\mathrm{C}$ detonation first burns the material along the equator and reaches the center, and then it burns the matter along the equator. There is more time for the matter to move outward and expand, thus yielding a weaker heating effect.

The IMEs form the middle layer, from 7000 to $17,000 \mathrm{~km}$ $\mathrm{s}^{-1}$ along the poles and from 12,000 to $15,000 \mathrm{~km} \mathrm{~s}^{-1}$ along the equator. Along the poles, as the velocity increases, which corresponds to lower-density matter, some unburned ${ }^{12} \mathrm{C}$ can be seen. However, there is no such trace along the equator. Again, this demonstrates that the detonation along the poles is weaker than that along the equator due to the time lapse during expansion. 
From 17,000 to $21,000 \mathrm{~km} \mathrm{~s}^{-1}$ along the poles, ${ }^{56} \mathrm{Ni}$ dominates the ejecta again. These are the products of the $\mathrm{He}$ detonation, as the composition is mostly ${ }^{4} \mathrm{He}$. They share similar mass fractions up to the surface. On the other hand, along the equator, there is more ${ }^{56} \mathrm{Ni}$ from 15,000 to $19,000 \mathrm{~km}$ $\mathrm{s}^{-1}$ and more ${ }^{4} \mathrm{He}$ from $19,000 \mathrm{~km} \mathrm{~s}^{-1}$ onward.

\subsubsection{Ring-type Explosion}

The one-ring configuration shows a stronger explosion than the one-bubble counterpart through shock convergence near the poles. The explosion is also stronger with higher ${ }^{56} \mathrm{Ni}$ production. In the bottom left and right panels of Figure 5, we plot the same as the top panels but for model 100-100-R.

Along the poles, the ${ }^{56} \mathrm{Ni}$ builds the core of the iron-peak elements that extends up to $12,000 \mathrm{~km} \mathrm{~s}^{-1}$. Other iron-peak elements, such as ${ }^{57} \mathrm{Ni}$ and ${ }^{58} \mathrm{Ni}$, are overwhelmed by the IMEs $\left({ }^{28} \mathrm{Si}\right.$ and ${ }^{32} \mathrm{~S}$ as two representatives) at a lower velocity of $10,000 \mathrm{~km} \mathrm{~s}^{-1}$. Beyond 12,000 $\mathrm{km} \mathrm{s}^{-1}$, IMEs are the dominant species until the surface. A minor jump of ${ }^{56} \mathrm{Ni}$ can be seen only near the surface, unlike model $100-100-\mathrm{B}$. The ${ }^{4} \mathrm{He}$ is also much lower than the IMEs and is $\sim 20 \%$ of the surface abundance.

On the other hand, there is no such transition of the ${ }^{56} \mathrm{Ni}$-rich core to the IME envelope along the equator. A rapid jump of IMEs appears near $9000 \mathrm{~km} \mathrm{~s}^{-1}$. However, the total abundance is about $0.1-0.2$ lower than ${ }^{56} \mathrm{Ni}$.

\subsubsection{Model Comparison}

Models 100-100-B and 100-100-R constitute two extremes of initial detonation configuration with minimal perturbation from the spherical detonation. The distribution of elements in the velocity space appears to be very distinctive.

Without sufficient shock convergence, the explosion ejecta consists of much stronger traces of IMEs in the middle layer and ${ }^{56} \mathrm{Ni}-{ }^{4} \mathrm{He}$ transition near the surface. The distribution of ${ }^{56} \mathrm{Ni}$ is discontinuous along some direction in the B-type model but continuous in the R-type model. Meanwhile, IMEs are more pronounced in the R-type model near the surface but not the B-type model. These distinctive features can be the indicators of where the initial He detonation starts.

\section{5. ${ }^{56}$ Ni Mass: Dependence on the WD Mass and Detonation Morphologys}

The explosion of a sub-Chandrasekhar-mass WD is known to be sensitive to the initial WD mass, $M$. This is because the WD is degenerate, so the central density varies largely from $10^{7} \mathrm{~g} \mathrm{~cm}^{-3}$ (for $M \sim 0.95 M_{\odot}$ ) to $10^{9} \mathrm{~g} \mathrm{~cm}^{-3}$ (for $M \sim$ $\left.1.20 M_{\odot}\right)$. The difference in the central density corresponds to a difference in the average density of WD matter, where the $\mathrm{CO}$ matter can be completely burned to ${ }^{56} \mathrm{Ni}$ when it has a typical density of $\sim 5 \times 10^{7} \mathrm{~g} \mathrm{~cm}^{-3}$. As a result, the amount of final ${ }^{56} \mathrm{Ni}$ drastically varies by a factor of $2-6$ when $M$ increases from 0.9 to $1.2 M_{\odot}$.

In Figure 7, we show the final ${ }^{56} \mathrm{Ni}$ mass against $M$ for the four different detonation morphologies described in the previous subsections. Here the magenta box represents the range of ${ }^{56} \mathrm{Ni}$ derived from the gamma-ray data from SN 2014J. See Section 4 for the application to SN 2014J. Models in which the $\mathrm{C}$ detonation cannot be triggered are omitted, i.e., 095-050B, 095-050-B, 100-050-B, 105-050-B, 110-050-B, and 095050-D. All of these show a typical He nova event without the $\mathrm{C}$ detonation (Kippenhahn \& Thomas 1978; Piro \& Bildsten 2004), which is inconsistent with the supernova observation that the whole star is disrupted after the explosion. From both figures, we can see that the $\mathrm{S}$ series is the strongest, and then the $\mathrm{D}$ and $\mathrm{R}$ series. The $\mathrm{B}$ series is the weakest for the same initial $M$.

The possibility of triggering the $\mathrm{C}$ detonation in the low $M_{\mathrm{He}}$ limit relies on the detonation symmetry. The CO WD models with a lower mass from $M=0.95$ to $1.10 M_{\odot}$ show that with the lowest symmetry (B type), there is no geometric convergence. The only shock collision occurs when the detonation reaches the equator of the WD. The assumed boundary condition (reflection symmetry) allows the arriving shock waves to collide in a laminar way. As indicated in Paper II, when $M$ increases, the minimum $M_{\mathrm{He}}$ required for triggering the second detonation decreases. According to similar work without assuming reflection symmetry (e.g., Fink et al. 2014), the minimum necessary $M_{\mathrm{He}}$ drops from 0.126 to $0.0035 M_{\odot}$ when the CO WD mass increases from 0.810 to $1.385 M_{\odot}$. Both works give us an insight that a higher $M_{\mathrm{He}}$ is necessary to provide sufficient shock strength in triggering the $\mathrm{C}$ detonation.

By only considering the WD mass, a higher $M$ means that the transition from the $\mathrm{CO}$ core to the He envelopes takes place at a higher density. This increases the typical reaction rate and hence the energy production. The postshock temperature in the He envelope is, therefore, higher for more massive WDs, where the burned matter can reach the threshold temperature easier, independent of additional geometrical convergence. Thus, a higher- $M$ model favors the trigger of the second detonation.

We recall that in simulations using a hemisphere of a WD, the dependence on the $M_{\mathrm{He}}$ is stronger than in simulations using a quadrant. This is because the detonation starts from one pole, and then the detonation wave wraps over the He envelope and converges at the other pole. In this situation, a shock convergence similar to the R-type detonation always happens. As shown in the table for the R-type detonation, the corresponding minimum $M_{\mathrm{He}}$ for the second detonation is lower.

For D, R, and S, they have a higher symmetry where there is geometric convergence by means of oblique shock, twodimensional shock convergence (from a ring to a point), and three-dimensional shock convergence (from a sphere to a point), respectively. The resultant temperature in the $\mathrm{CO}$ core can be much enhanced by the converged shock strength. Therefore, the minimum $M_{\mathrm{He}}$ required to trigger the second detonation is more relaxed.

The difference in the final ${ }^{56} \mathrm{Ni}$ mass for the same $M$ at different $\mathrm{He}$ detonation is related to the propagation of the C-detonation direction. The $\mathrm{S}$ model is always the strongest because the $\mathrm{C}$ detonation begins at the center and propagates outward, so that most of the star remains approximately static before the detonation wave arrives. This ensures that the matter remains less expanded and hence maintains a higher density, which results in a stronger explosion. On the contrary, in the B, $\mathrm{D}$, and $\mathrm{R}$ models, the off-center $\mathrm{C}$ detonation means that the $\mathrm{C}$ detonation has to overcome the density gradient in order to reach the high-density matter in the core. This implies that the relative explosion strength is weaker because of the density gradient. 


\subsection{Morphology of Remnants}

The early ${ }^{56} \mathrm{Ni}$ in the ejecta near the surface provides distinctive hints of the asphericity of the detonation model. Furthermore, the distribution of iron-peak elements, which are shock-heated by the backward shock in the ejecta, can reach reach a temperature of $\sim 10^{6} \mathrm{~K}$, which is sufficiently hot for $\mathrm{X}$-ray emission for further diagnosis. This will give further constraints on the explosion models. For example, the X-ray spectra of the SNR 3C 397 have been used as a diagnosis of a Chandrasekhar-mass WD progenitor (Yamaguchi et al. 2015; Dave et al. 2017; Leung \& Nomoto 2018).

In Figure 6, we plot the element distribution of $\mathrm{Cr}, \mathrm{Mn}, \mathrm{Fe}$, and $\mathrm{Ni}$ for models 100-100-B, 100-100-R, 100-100-D, and 100-100-S. We assume that after the star reaches homologous expansion, the distribution of ejecta remains mostly unchanged. However, we also remark that the radiation energy during the decay of radioactive isotopes can still trigger inner motion and affect the element distribution. But the effect is secondary compared to the initial distribution during explosion.

The distributions of the iron-peak elements show more diversity than the major elements, as shown in the previous section. From the contour shape, we observe two features. First, $\mathrm{Cr}$ and $\mathrm{Mn}$ almost follow each other, and $\mathrm{Fe}$ and Ni follow each other. Second, $\mathrm{Cr}$ and $\mathrm{Mn}$ tend to have a more spherical distribution, while $\mathrm{Fe}$ and Ni follow the detonation geometry more closely.

Model 100-100-R shows the largest deviation from a spherical structure among all four elements. Model 100-100$\mathrm{B}$ has spherical $\mathrm{Cr}$ and $\mathrm{Mn}$ but aspherical $\mathrm{Fe}$ and Ni. Similar characteristics appear for model 100-100-D. All elements are spherical in model 100-100-S, as anticipated by the initial spherical symmetry. To distinguish between models 100-100-B and $100-100-\mathrm{D}$, we notice that the distribution of $\mathrm{Cr}$ is more irregular in model 100-100-D compared to the quasi-spherical distribution in model 100-100-B. However, the difference is subtle.

\section{Case Study: Application to SN 2014J}

\subsection{The Inspiring Case of SN 2014J}

The closest SN Ia in the last four decades, SN 2014J is a special example of SNe Ia exploded in the nearby galaxy M82, just $3.3 \mathrm{Mpc}$ away. Its closeness to the Milky Way has provided the chance for detailed multiband observations, including the radio (Pérez-Torres et al. 2014), infrared, optical (Goobar et al. 2014; Kawabata et al. 2014), UV (Foley et al. 2014), X-ray (Terada et al. 2016), and gamma-ray (Diehl et al. 2014; Churazov et al. 2015; Diehl et al. 2015; Diehl 2015; Siegert \& Diehl 2015; Isern et al. 2016) bands, with its spectra at early and late times (Ashall et al. 2014; Jack et al. 2015; Dhawan et al. 2018).

Measurements of SN 2014J have been made in different works in the literature. In Churazov et al. (2014), the estimated $M^{56}{ }_{\mathrm{Ni}}=0.56 \pm_{0.06}^{0.14} M_{\odot}$, and the estimated ejected mass is $1.2 \pm_{0.5}^{1.9} M_{\odot}$. A similar measurement is found in Diehl et al. (2015), who gave $M^{56} \mathrm{Ni}=0.50 \pm 0.12 M_{\odot}$. For ${ }^{57} \mathrm{Ni}$, Yang et al. (2018) reported that ${ }^{57} \mathrm{Ni} /{ }^{56} \mathrm{Ni}$ has a mass ratio of $0.065 \pm_{0.004}^{0.005}$ based on the $B$-band maximum light and $0.066 \pm_{0.008}^{0.009}$ based on the pseudobolometric light curve. Stable $\mathrm{Ni}$ mass is constrained at $0.053 \pm 0.018 M_{\odot}$ (Dhawan et al. 2018).
It shows gamma-ray features that agree well with the classical spherical pure deflagration model W7 (Churazov et al. 2014; Diehl et al. 2015). In photometry, SN 2014J appears to be a normal SN Ia (Isern et al. 2016) that shows a comparable structure with the W7 model (Nomoto et al. 1984) and a similar abundance profile as the heavy elements (e.g., $\left.{ }^{56} \mathrm{Ni}\right)$ in the core and lighter elements (e.g., Si and $\mathrm{S}$ ) in the envelope (Ashall et al. 2014).

However, detailed examinations of the observational data of SN 2014J reveal some differences from ordinary SNe Ia. For example, the rise of the UVOIR light curve with time (e.g., Nugent et al. 2011) shows its delay in SN 2014J. The early light curve of SN 2014J suggests a "shoulder" only a few days after the first light (Goobar et al. 2014). The late-time evolution (beyond a few hundred days) shows derivations from classical SNe Ia such as SN 2011fe, where the slower decline rate suggests interactions with CSM (Foley et al. 2014; Yang et al. 2018). The ultraviolet data of SN 2014J show large extinction (Brown et al. 2015). Such extinction and CSM can be in the dusk disk structure (Nagao et al. 2017). Images around SN 2014J do not show an observable companion star, thus making its companion as a red giant unlikely (Kelly et al. 2014; Margutti et al. 2014; Pérez-Torres et al. 2014).

The progenitor of SN 2014J is still a question of debate (e.g., Margutti et al. 2014; Pérez-Torres et al. 2014; Dragulin \& Hoeflich 2016; Graur \& Woods 2019). We note that these constraints on CSM cannot be applied to the pre-SN environment of a uniformly rotating WD with a slightly super-Chandrasekhar mass in the single-degenerate scenario, as calculated by Benvenuto et al. (2015). The gamma-ray signal cannot distinguish with high significance which class SN 2014J belongs to (Terada et al. 2016).

\subsection{Aspherical Features of SN 2014J and Constraints on Models}

According to further examinations, the observational data of SN 2014J show features that deviate from the spherical approximation as discussed below.

First, the early gamma-ray observations with INTEGRAL discovered the lines at 158 and $812 \mathrm{keV}\left(T_{1 / 2}=6.6\right.$ days) that are characteristic for the ${ }^{56} \mathrm{Ni}$ decay around 17.5 days after the inferred explosion date (Diehl et al. 2014; Isern et al. 2016). The model fits of Isern et al. (2016) appeared more consistent with a redshifted and broadened ${ }^{56} \mathrm{Ni}$ emission, so they suggested ejection of ${ }^{56} \mathrm{Ni}$-rich material in a blob moving away from the observer. The analysis by Diehl et al. (2014) was performed in finer energy bins and without any model bias; their sampling of possible spectral solutions suggests a narrow emission line from ${ }^{56} \mathrm{Ni}$ at the laboratory energy value, with indications of blue- as well as redshifted satellite. This led them to suggest a model with ${ }^{56} \mathrm{Ni}$ ejected perpendicular to the observer's line of sight. But the total significance of this surface ${ }^{56} \mathrm{Ni}$ line emission is only $3 \sigma-$ $4 \sigma$; hence, both interpretations remain possible.

In any case, the existence of ${ }^{56} \mathrm{Ni}$ near the surface is witnessed by the INTEGRAL data and is the main motivation for our study. The missing $\mathrm{C}$ and $\mathrm{O}$ absorption lines are possibly connected to the exposed ${ }^{56} \mathrm{Ni}$ (Goobar et al. 2014). Also, expected Ni lines are however not seen in the infrared at this time.

The second aspherical feature appears at later times. When the energy output is dominated by the decay of ${ }^{56} \mathrm{Co}\left(T_{1 / 2}=77.1\right.$ days), the measured Doppler shifts of the ${ }^{56} \mathrm{Co}$ decay lines show 

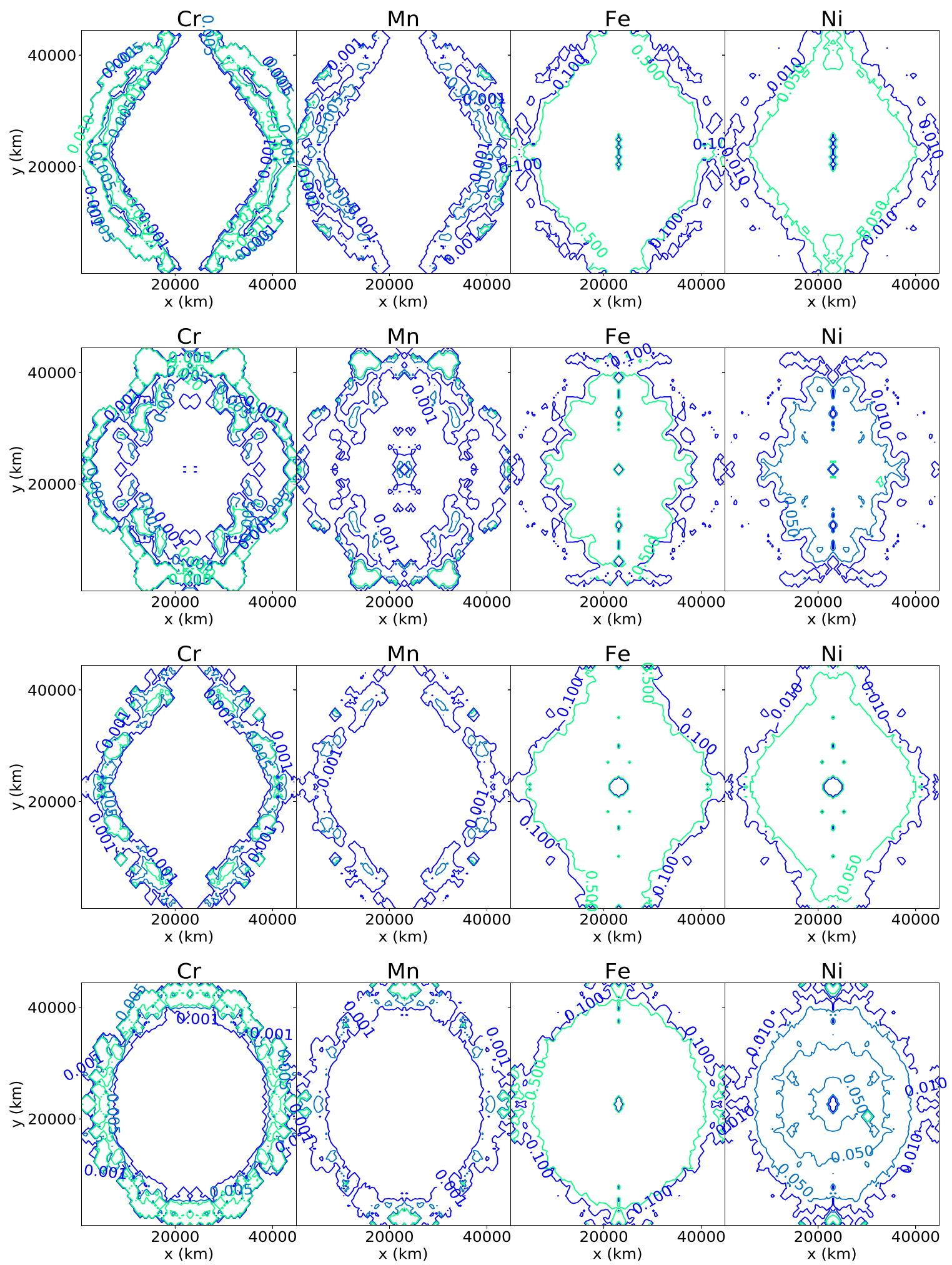

Figure 6. Stable element mass fraction distributions of the explosion models 100-100-B (first row), 100-100-R (second row), 100-100-D (third row), and 100-100-S (fourth row) for $\mathrm{Cr}, \mathrm{Mn}, \mathrm{Fe}$, and $\mathrm{Ni}$ (left to right panels). The numbers indicate the corresponding mass fraction contours for these isotopes.

the expected behavior, plus an additional structure of (at least) three blobs of distinctive velocities (i.e., showing an early blueshift when the redshifted part is opaque in the line of sight and then becoming symmetric with no Doppler shift later, as pointed out in Diehl et al. 2015). Instead, at least three distinctive centroid energies could be identified. This may correspond to fluid parcels containing ${ }^{56} \mathrm{Co}$ being ejected with different velocities with respect to the observer's frame. This timedependent variation of the Co decay line frequency, denoted as "flickering," is observed in SNe Ia for the first time. This suggests the possibility that "blobs," or large-scale asymmetries, developed during the explosion. 
To summarize, SN 2014J has shown conflicts with the classical spherical model. These include (1) the observed Doppler-broadened ${ }^{56} \mathrm{Co}$ lines, which have an irregular appearance with time (Diehl et al. 2015); (2) hints of the ${ }^{56} \mathrm{Ni}$ decay lines on the surface (Diehl et al. 2014); and (3) an enhanced ionization on the outer part of the star, as revealed by the early atomic line spectra.

\subsection{Near-Chandrasekhar Mass Models that Produce Surface ${ }^{56} \mathrm{Ni}$}

Among the above mentioned features of SN 2014J, the existence of ${ }^{56} \mathrm{Ni}$ near the surface suggested by the INTEGRAL data is the main motivation for the present study.

Before discussing the sub-Chandrasekhar mass models in the following subsections, we note that near-Chandrasekhar mass models have variations depending mainly on the mass accretion rate, and some models produce ${ }^{56} \mathrm{Ni}$ near the surface of WDs. In the classical picture of the near-Chandrasekhar mass model, ${ }^{56} \mathrm{Ni}$ is concentrated in the inner core (The \& Burrows 2014). However, ${ }^{56} \mathrm{Ni}$ is produced near the surface in the following models.

Nomoto (1982b) showed several models where the WD mass increases to the near-Chandrasekhar mass with slow accretion of He. If the accretion rate is low enough, the accreted $\mathrm{He}$ is too cold to be ignited, thus being just accumulated near the surface. Eventually, the WD becomes massive enough to ignite the central C-deflagration.

In the late detonation model by Yamaoka (1992), the deflagration-detonation transition can occur in the outer layer of the near-Chandrasekhar mass WD and burns He to produce ${ }^{56} \mathrm{Ni}$ near the surface (see model W7DHE in Figure 3 of Yamaoka 1992). Such a deflagration-detonation transition might occur at the very steep density gradient near the WD surface even with a small amount of He. The deflagration-detonation transition near the surface would be likely to occur in an aspherical manner which might be interesting for further study.

\subsection{Modeling Issues on the Sub-Chandrasekhar WD Models for SN 2014J}

Although the production of ${ }^{56} \mathrm{Ni}$ near the surface is possible in the late detonation of the near-Chandrasekhar-mass model (W7DHE), here we focus on the sub-Chandrasekhar-mass models in order to apply the results of this Paper IV to the "asphericity", of SN 2014J.

The object SN 2014J was first proposed to be the ignition of an "He belt" accumulated in the orbital plane of the binary (Diehl et al. 2014). The He-belt model was motivated by the early ${ }^{56} \mathrm{Ni}$ decay line detected with a close-to-zero line Doppler shift. This picture can explain the origin of the early-time ${ }^{56} \mathrm{Ni}$ decay line. However, on top of that, in Diehl et al. (2014), such a decay line has a very small redshift. The He-belt model ejects $\mathrm{Ni}$ synthesized in the He envelope along the equator direction. ${ }^{8}$ As a result, it provides the source of $\mathrm{Ni}$ with a small Doppler shift. But, it remains unclear if the He belt can be formed and stably maintained during the binary accretion (Kippenhahn \& Thomas 1978; Piro \& Bildsten 2004).

In the following discussion, we search for a qualitative model that may resemble the observed characteristics of SN 2014J. We note that the model asphericity should not be too

\footnotetext{
8 Notice that in general, a static WD is considered, and "poles" has a graphic meaning of being the upper and lower ends of the sphere, while "equator" means the symmetry plane between the two poles.
}

strong, as otherwise it would violate the similarity of SN 2014J with the classical spherical model. Thus we set the He envelope in spherical form. To generate the required surface $\mathrm{Ni}$, different He-detonation configurations are studied.

Our attempt is to elucidate the physical conditions that are able to qualitatively reproduce the distinctive features of SN 2014J. Owing to the complexity of multiple data constraints and the subtlety in the interpretations of different observations, we avoid scrutinizing an exact or complete model that can explain all of the quantitative features for SN 2014J. Our focus is, therefore, on the early ${ }^{56} \mathrm{Ni}$ line emission, the ${ }^{56} \mathrm{Ni}$ mass as derived from the peak ( $V$-band) luminosity, large-scale asymmetries as implied by the flickering ${ }^{56} \mathrm{Co}$ decay line profiles, and the ${ }^{57} \mathrm{Ni} /{ }^{56} \mathrm{Ni}$ mass fraction ratio from the latetime light curve. Using ${ }^{57} \mathrm{Ni} /{ }^{56} \mathrm{Ni}$ to constrain the $\mathrm{SN}$ explosion models as proposed in Seitenzahl et al. (2009) has been done in other SNe Ia, for example, SN 2012cg.

To recapitulate, based on the two-dimensional models, we aim at searching for (1) WD parameters that correspond to the general features of SN 2014J and (2) appropriate He-detonation triggers that produce the global asymmetries and qualitative features of SN 2014J, including large-scale asymmetry and/or near-surface production of ${ }^{56} \mathrm{Ni}$.

\subsection{Constraints on Progenitor WD Mass}

The ${ }^{56} \mathrm{Ni}$ mass observed in SN 2014J provides the important constraint on the progenitor WD mass. In Diehl (2015), ${ }^{9}$ the ${ }^{56} \mathrm{Ni}$ mass is estimated to be $0.49 \pm 0.10 M_{\odot}$. From our simulation results in Table 1 , we identify the possible mass range to be $M=1.0-1.1 M_{\odot}$.

To account for the observed ${ }^{56} \mathrm{Ni}$ mass in SN 2014J shown by the magenta box in Figure 7, we require $M=0.95-1.00 M_{\odot}$ for the "S"-series and $M=1.00-1.10 M_{\odot}$ for the "B"-series $\left(M_{\mathrm{He}}=0.10 M_{\odot}\right)$. "D"- and "R"'-series require $M=1.05-1.10$ (1.00-1.05) $M_{\odot}$ for $M_{\mathrm{He}}=0.05(0.10) M_{\odot}$.

\subsection{Constraints on Explosion Mechanisms}

Another aspect by which to constrain SN 2014J is the explosion geometry. The gamma-ray signal of SN 2014J has suggested the (near-)surface ${ }^{56} \mathrm{Ni}$. We examine which initial detonation geometry allows the formation of ${ }^{56} \mathrm{Ni}$ at these regions. To extract the final distribution of ${ }^{56} \mathrm{Ni}$, we use the tracer particle data when the ejecta reaches homologous expansion and the structure of the ejecta is frozen out.

Future observations of SN 2014J, which will be able to disentangle the morphology, may thus provide clues to the initial configuration from measurements of different elements. For example, in Grefenstette et al. (2014, 2017), such a technique is pioneered in showing ${ }^{44} \mathrm{Ti}$ in Cas A to demonstrate how to disentangle and map the gamma rays.

In Figure 3, we show the distributions of some representative elements for models with different detonation geometry. For example, for models starting with an He-detonation bubble, the $\mathrm{Ni}$ and $\mathrm{Si}$ ejecta are in a cocoon shape, compared to the spherical shape in the spherical detonation. The B type is also highly distinctive due to the thick layer of O-rich ejecta, compared to the spherical counterpart. On the other hand, the D and $\mathrm{R}$ types are different from the other two by the quasispherical O- and Si-rich ejecta, while the Ni ejecta maintain an

\footnotetext{
9 Other works in the literature give a similar range. For example in Churazov (2014) the upper limit can reach $0.7 M_{\odot}$, but the uncertainties are similar.
} 

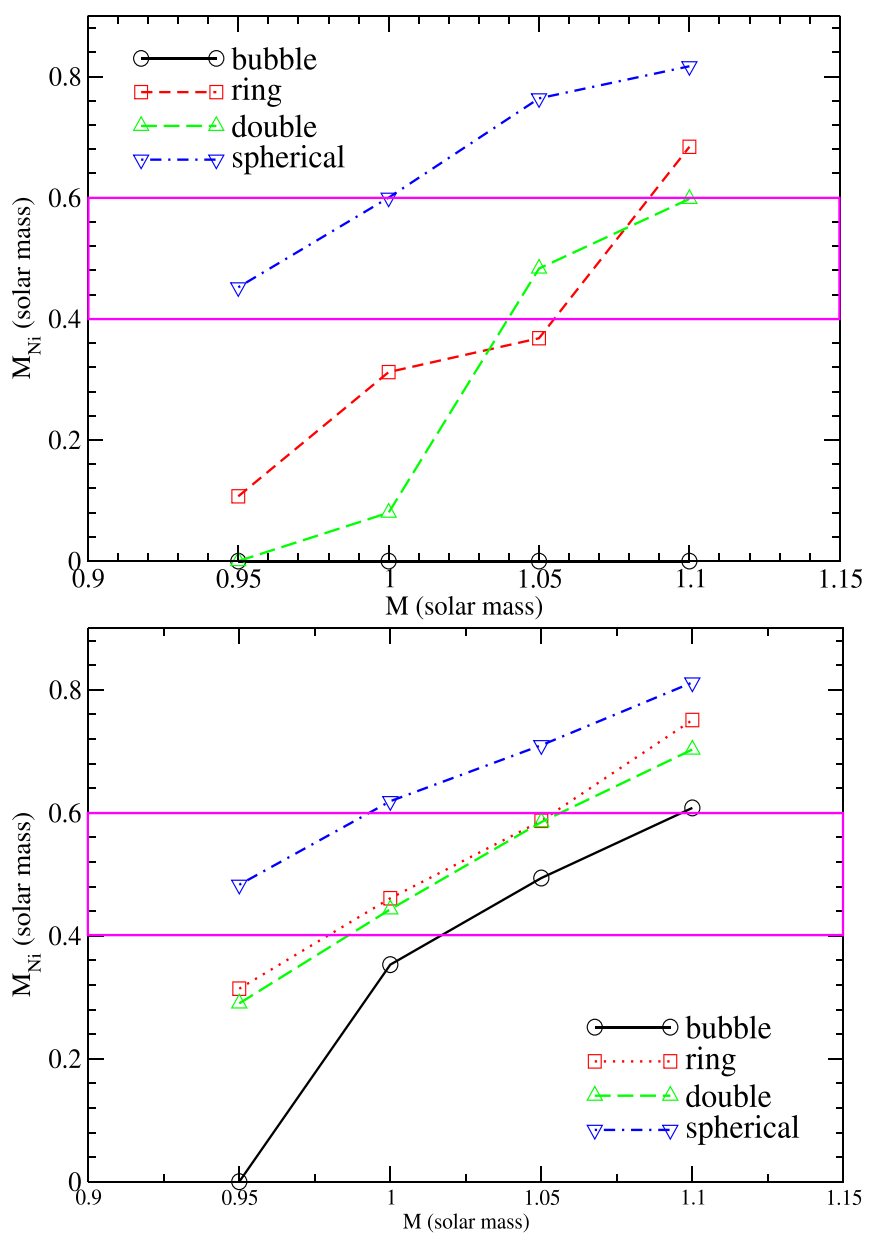

Figure 7. The ${ }^{56} \mathrm{Ni}$ mass against $M$ for the four different initial He-detonation configurations for $M_{\mathrm{He}}=0.05 M_{\odot}$ (upper panel) and $0.10 M_{\odot}$ (lower panel). The magenta box represents the range of ${ }^{56} \mathrm{Ni}$ derived from the gamma-ray data from SN 2014J.

observable ellipticity. However, between the D- and R-type models, $\mathrm{Si}$ and Ni show a similar distribution.

\subsection{Constraints on the He-envelope Mass}

We now examine the dependence of the large-scale asymmetry on the He-envelope mass. In Paper II, we presented a parameter survey on the nucleosynthesis yield of SNe Ia using the sub-Chandrasekhar-mass WD as the initial progenitor. It is shown that the He-envelope mass can strongly enhance the production of some Fe-peak isotopes, including ${ }^{48} \mathrm{Ti},{ }^{50,51} \mathrm{~V}$, and ${ }^{52} \mathrm{Cr}$.

In Figure 8, we plot the ${ }^{56} \mathrm{Ni}$ distribution for models 100 $050-\mathrm{R}$ and 100-100-R. They differ by the mass of the He envelope from 0.05 to $0.1 M_{\odot}$. The distribution of ${ }^{56} \mathrm{Ni}$ after the explosion can show the existence of large-scale asymmetry. In model 100-100-R, with a more massive He envelope, the He detonation is strong enough to drive the inward propagation of the detonation to the core; this results in complete disruption, where most of the matter in the CO core is spherical. Therefore, this scenario will be less likely to exhibit a flickering, as seen in SN 2014J. However, its ${ }^{56} \mathrm{Ni}$ also distributes close to the surface, which is an important feature of SN 2014J. As a result, the ejected ${ }^{56} \mathrm{Ni}$ around $10^{\circ}$ from the rotation axis has a higher radial velocity. The lower He-envelope mass makes the production of ${ }^{56} \mathrm{Ni}$ lower, and the synthesized ${ }^{56} \mathrm{Ni}$ is covered by the original He envelope, which can block the gamma rays emitted by the radioactive decay. This means that a lower- $M_{\mathrm{He}}$ model has more difficulty reproducing the early gamma rays observed from SN 2014J.

Following the expansion of the ejecta, when the matter becomes optically thin to gamma rays, the inner structure of the ${ }^{56} \mathrm{Ni}$ and ${ }^{56} \mathrm{Co}$ distribution will be exposed. The velocity fluctuation, as seen from Figure 8, depending on the ejecta angle and the exact time to become optically thin, may coincide with the flickering feature, as seen in SN 2014J.

\subsection{Constraints from Nucleosynthesis}

In Figure 9, we plot the ${ }^{57} \mathrm{Ni}$ and ${ }^{56} \mathrm{Ni}$ yields (prior to decay) of our simulated SN Ia models. Shown are models for $M_{\mathrm{He}}=0.05$ (solid lines) and 0.10 (dashed lines) at solar metallicity. We also show the SN 2014J data (crosses; Yang et al. 2018). Apparently, the models listed there are not sufficient to explain the high ${ }^{57} \mathrm{Ni}$ value of this $\mathrm{SN}$.

Similarly, to demonstrate the effects of metallicity, we plot in the figure a red dashed-double-dotted line for model 110-050$\mathrm{B}$ at $0,1,3$, and $5 Z_{\odot}$ (corresponding to the data points from the bottom to the top). To be consistent with the SN 2014J data, a model $Z=\sim 5 Z_{\odot}$ is required. All of the trend lines for other models behave similarly. In Paper I, we have showed that the metallicity has a minor impact on the global energetic explosion. Instead, it shows its influence on the relative abundance ratio, in particular the high- $Y_{e}$ isotopes including ${ }^{54} \mathrm{Fe},{ }^{58} \mathrm{Ni}$, and ${ }^{55} \mathrm{Mn}$.

From this figure, we observe that in order to explain the abundance pattern of SN 2014J, a high-metallicity model $\gtrsim 4 Z_{\odot}$ is necessary to be consistent with the high ${ }^{57} \mathrm{Ni}$ mass relative to ${ }^{56} \mathrm{Ni}$.

\section{Extension to Other SN Observations}

\subsection{Remnant Morphology}

The morphology of the SN ejecta and the shape of the SNR, such as Tycho (Ferrand et al. 2019), may directly link to the initial explosion configuration. By tracing the line emission of shock-heated ejecta, the abundance of the measured elements can be revealed. In Seitenzahl et al. (2019), the tomography of three very young SNRs, 0519-69.0, 0509-67.5, and N103B, in the Large Magellanic Cloud (Hughes et al. 1995) are studied for the first time. By examining the S XII, Fe IX, and Fe XV lines, they reconstructed the large-scale distributions of these elements in these remnants. These objects are sufficiently young such that the shocked-heated matter remains clearly visible and the shock front has not completely swept through the remnant.

The SNR 0519-69.0 has a more spherical shape but with smallscale perturbations on the surface, as depicted by the X-rays. From our simulations, such features are possible when we consider the Rayleigh-Taylor instabilities that freeze out during the expansion of the ejecta. As discussed in Seitenzahl et al. (2019), this remnant can be fitted by a Chandrasekhar-mass WD of mass $1.4 M_{\odot}$. Their total Fe mass is about $0.4 M_{\odot}$. This belongs to the lower side of SN Ia production (Leung \& Nomoto 2018). Such features can be obtained for a higher-mass progenitor of $\sim 1.4 M_{\odot}$ with an initial central density $>3 \times 10^{9} \mathrm{~g} \mathrm{~cm}^{-3}$.

The SNR 0509-67.5 also has a spherical shape where the ejecta, including the X-ray emitting part, demonstrates a 

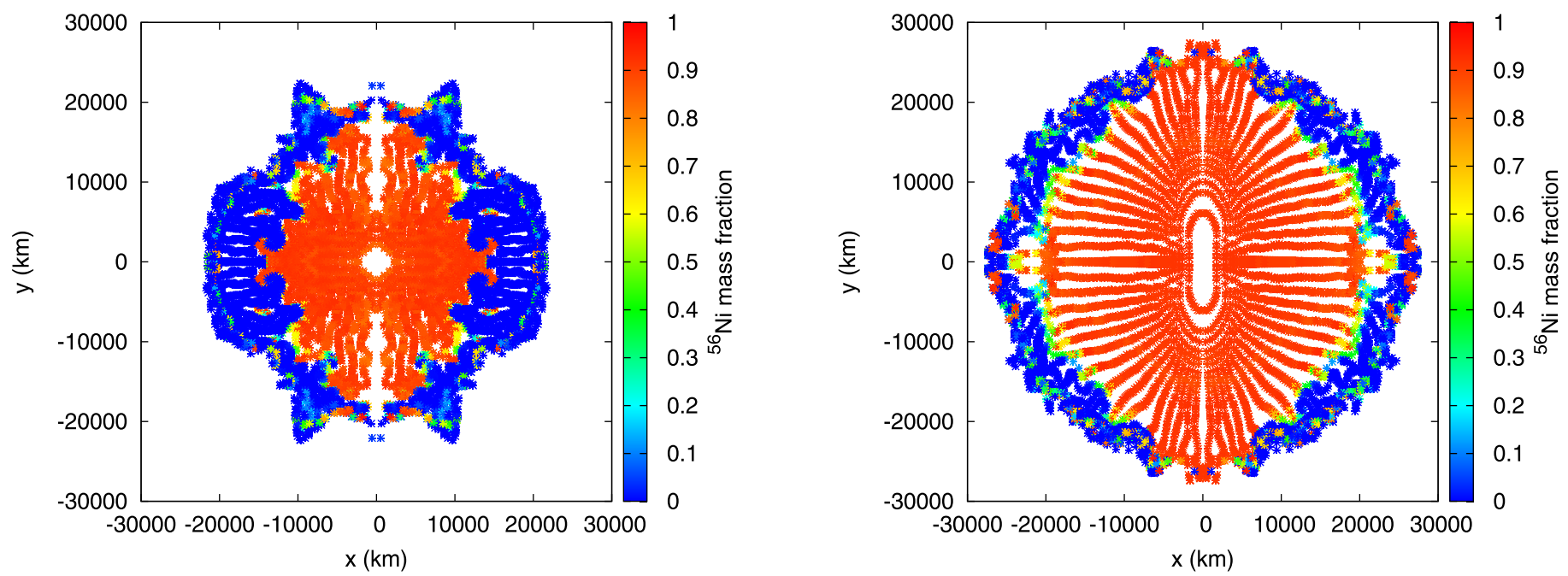

Figure 8. The ${ }^{56} \mathrm{Ni}$ mass fraction distribution of the explosion models 100-050-R (left panel) and 100-100-R (right panel).

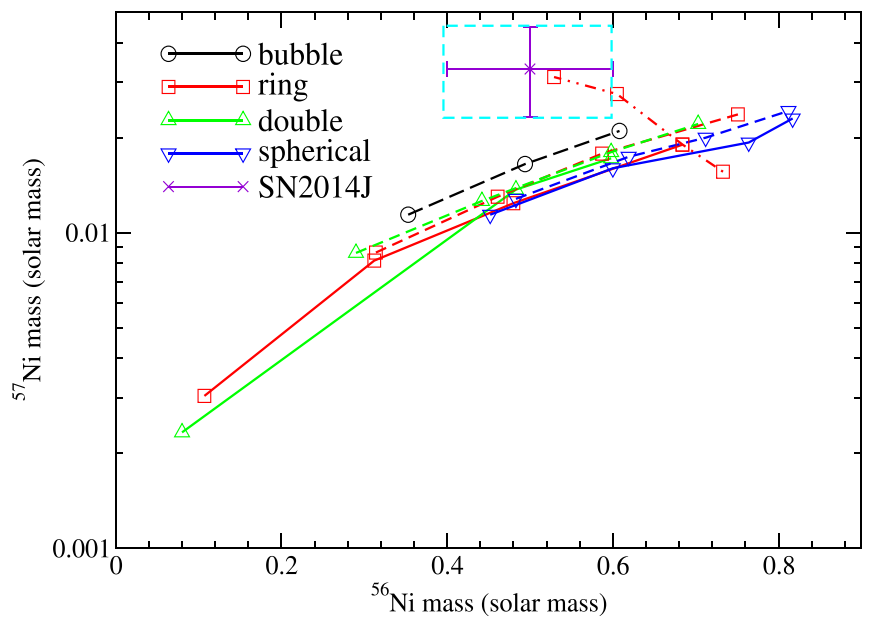

Figure 9. The ${ }^{57} \mathrm{Ni}$ mass against ${ }^{56} \mathrm{Ni}$ for the four different initial He-detonation configurations for $M_{\mathrm{He}}=0.05$ (solid line) and 0.10 (dashed line) $M_{\odot}$ and for solar metallicity. The data points show the observed ${ }^{57} \mathrm{Ni}$ against ${ }^{56} \mathrm{Ni}$ for different explosion models, including bubble (circle), ring (square), double (upward triangle) and spherical (downward triangle) model. The red dasheddouble-dotted line corresponds to the sequence of model 110-050-R50 for different initial metallicity $Z=0,1,3$, and $5 Z_{\odot}$ (from bottom to top). The data points from left to right correspond to the models with $M=0.9,1.0,1.05$, and $1.1 M_{\odot}$.

close-to-spherical structure. The estimated mass of this remnant is $\sim 1.0 M_{\odot}$ with $0.15 M_{\odot}$ He. To achieve such a spherical shape, a spherical He detonation is necessary avoiding any large-scale asymmetry created during the $\mathrm{C}$ detonation. In fact, when such a heavy He envelope is included, spherical detonation is preferred because the expected nuclear runaway time can easily be shorter than the convection timescale, thus triggering simultaneous burning in the spherical layer near the $\mathrm{He}-\mathrm{CO}$ interface. Also, as discussed in Paper II, the thick He envelope $>0.1 M_{\odot}$ can lead to a very severe excess in light iron-peaked elements such as Ti, V, and Cr. Future detection of these elements may provide further confirmation of this explosion picture. At last, its total Fe mass, $\sim 0.5 M_{\odot}$, can be mapped consistently with our models, such as 100-100-S.
The SNR N103B also has an aspherical ejecta shape. However, not much analysis of this object is reported in Seitenzahl et al. (2019). Despite that, from the morphology of the ejecta, an arrow or cone shape of Fe XIV distribution can be observed. Such an aspherical shape with a pointing effect can indicate the focused shock in the $\mathrm{CO}$ core and its consecutive breakout, as hinted from Figure 2. In our results, due to the reflection symmetry, the cone shape is always paired on both sides. We expect that, if we allow a single bubble without assuming reflection symmetry, a one-sided feature can emerge, as in more general simulations such as Tanikawa et al. (2019) and Gronow et al. (2020).

\subsection{Remnant Element Abundance}

Another possibility for tracing the remnant progenitor is to examine the abundance patterns. As described in previous sections, the X-ray spectra from SNRs can provide essential clues to the relative amount of elements, especially iron-peaked elements including $\mathrm{Cr}, \mathrm{Mn}, \mathrm{Fe}$, and Ni (Yamaguchi et al. 2015; Martínez-Rodríguez et al. 2017). They can directly constrain the type of WD progenitor and the explosion physics. In their work, the SNR spectra, including Tycho, Kepler, 3C 397, and G337.2-0.7 in the Milky Way and N103B in the Large Magellanic Cloud, are analyzed. It is suggested that, to explain the variety of remnant chemical abundance patterns, a factor beyond neutronization (i.e., metallicity tracer by ${ }^{22} \mathrm{Ne}$ ) is necessary. Here we examine how the metallicity affects the elemental distribution and ${ }^{56} \mathrm{Ni}$.

In Figure 10, we plot the mass and $Z$ dependence of ${ }^{56} \mathrm{Ni}$ production for the sub-Chandrasekhar-mass WD models with $M=0.9-1.2 M_{\odot}$ and $Z=0-0.04$. The ${ }^{56} \mathrm{Ni}$ production is strongly $M$-dependent and monotonically increasing with $M$. The transition from complete to incomplete burning shows the strongest effects between 0.9 and $1.0 M_{\odot}$ by a change of almost a factor of 10 within this mass range. The effects of metallicity can be seen but are small compared to the effects of $M$. In general, an $\sim 10 \%$ difference can be seen among the $Z$ range examined here. 


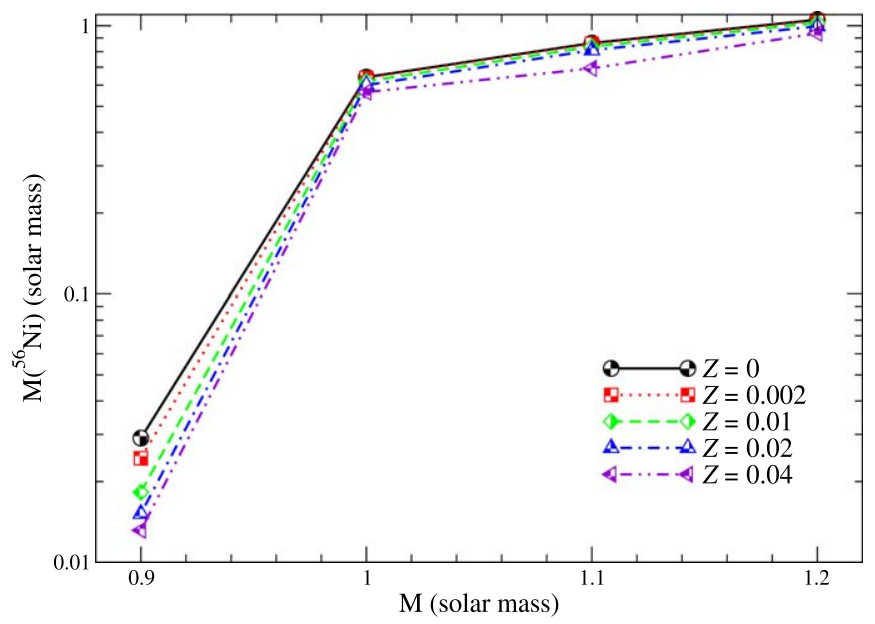

Figure 10. The ${ }^{56} \mathrm{Ni}$ production for the sub-Chandrasekhar-mass models with initial spherical detonation structure for $M=0.9-1.2 M_{\odot}$ at $Z=0,0.002$, $0.01,0.02$, and 0.04 .

In Figure 11, we plot the mass fraction ratio of $[\mathrm{Mn} / \mathrm{Fe}]$, $[\mathrm{Ni} / \mathrm{Fe}],[\mathrm{Cr} / \mathrm{Fe}]$, and $[\mathrm{Ca} / \mathrm{S}]$ for our sub-Chandrasekhar-mass WD models with a mass of $1.0 M_{\odot}$ for metallicity $Z=0$, $0.002,0.01,0.02$, and 0.04 . The metallicity effect is much larger. Major iron-peaked elements such as $[\mathrm{Mn} / \mathrm{Fe}]$ and $[\mathrm{Ni} /$ $\mathrm{Fe}]$ differ by $\sim 0.6$ dex between models with $Z=0$ and 0.04 . Both of them increase when $Z$ increases. On the other hand, minor iron-peaked elements such as $[\mathrm{Cr} / \mathrm{Fe}]$ and IMEs such as $[\mathrm{Ca} / \mathrm{S}]$ show much smaller variations by only $0.1 \mathrm{dex}$ in the $Z$ range examined. They decrease when $Z$ increases.

However, we remark that the exact values of $[\mathrm{Ca} / \mathrm{S}]$ and $[\mathrm{Cr} / \mathrm{Fe}]$ are more prone to systematic uncertainties. This is because the light iron-peaked elements, including $\mathrm{Ca}$ and $\mathrm{Cr}$, can be produced in both NSE and incomplete Si burning. For $\mathrm{Mn}, \mathrm{Fe}$, and $\mathrm{Ni}$, they are robustly produced in NSE matter, where complete and instantaneous energy release can be assumed. On the other hand, for $\mathrm{Ca}$ and $\mathrm{Cr}$, the numerical scheme of low-density burning can affect the abundance of these elements. For the same amount of energy release, a longer energy deposition time can slow down the thermal expansion. As a result, the matter has more time to carry out a slow nuclear reaction in the $\alpha$-rich freeze-out regime before the ejecta expands and becomes too cold for any significant nuclear reactions. The case for low-density matter contains complications because the actual reaction depends on the detailed chemical composition, which is not well traced in multidimensional composition. The simplified chemical composition (seven-isotope network) may not provide an accurate estimation of how fast those reactions and the associated energy production take place.

\section{Discussion}

\subsection{Connection to Works in the Literature}

Our work has suggested that He detonation is the key feature for explaining some well-observed SNe Ia, such as SN 2014J. However, it also remains to be understood in detail how the ignition of surface He can be coupled to the current explosion mechanism that centrally ignites carbon in the WD. Threedimensional low Mach number hydrodynamical simulations of the He-burning envelope are necessary for realizing how the

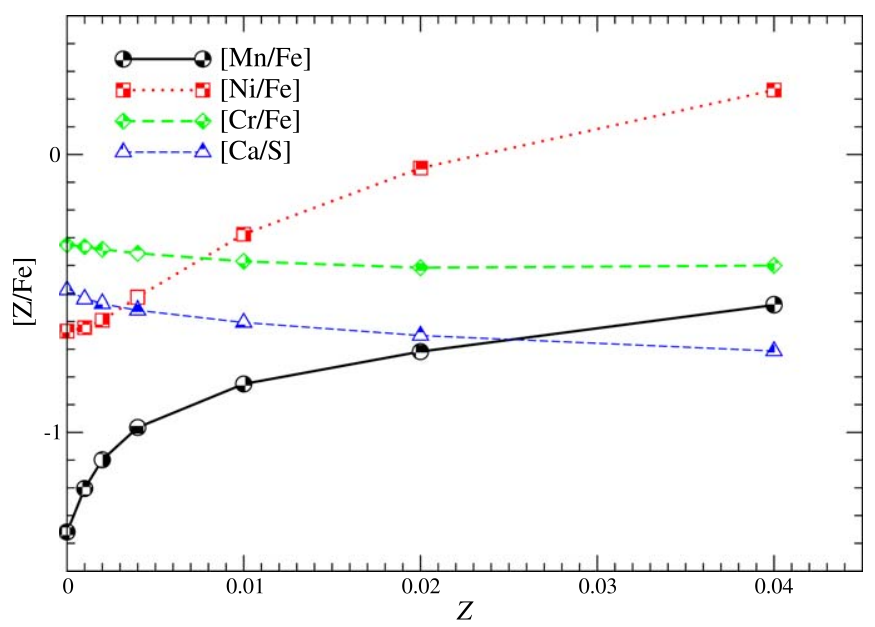

Figure 11. Abundance ratios for the sub-Chandrasekhar-mass model with initial spherical detonation structure for $M=1.0 M_{\odot}$ at $Z=0,0.002,0.01$, 0.02 , and 0.04 .

initial hydrostatic He burning develops into nuclear runaway. The detonation size is typically assumed to be as large as the pressure scale height (Bildsten et al. 2007; Shen \& Bildsten 2009; Dan et al. 2014). On the other hand, temperature fluctuations tend to favor the ignition at a single spot, which has a much smaller size. A stringent limit on the temperature fluctuation appears because of its small size (Holcomb et al. 2013). Contamination of C-rich matter from the $\mathrm{CO}$ core can be an alternative to decreasing the necessary size of nuclear burning (Shen \& Moore 2014).

The prerunaway phase of the double-detonation model was unclear until recent large-scale works on clarifying the possibility of such a proposal (Jacobs et al. 2016). Depending on the convection flow, different He-runaway pattern scenarios could occur from the most nonspherical extreme, i.e., a bubble, to the most spherically symmetric case. The modeling of such processes is typically much longer than the hydrodynamics timescale in order to directly capture the first nuclear runaway from nuclear reactions. To resolve the first runaway, the simulation requires the hydrodynamics timescale $t_{\text {hyd }}$ to be smaller than the timescale of nuclear burning $t_{\text {burn }}$ and convection $t_{\text {conv }}$, i.e., $t_{\text {hyd }}<t_{\text {burn }}<t_{\text {conv }}$ (Glasner et al. 2018). Only recently have there been a few pioneering models using hydrodynamics simulations in the low Mach number regime to follow how the convection develops into nuclear runaway selfconsistently (Zingale et al. 2013; Jacobs et al. 2016). Therefore, to trace the possible origin and explain the observations of SN 2014J, we considered different possibilities for how the $\mathrm{He}$ detonation can be triggered.

On the other hand, once a detonation spot forms, the second detonation is generally inevitable. Recent three-dimensional large-scale hydrodynamics simulations of one- or multiple-spot He detonation has been found to be robust in triggering the offcenter detonation by geometric convergence in a quiet $\mathrm{He}$ envelope (Moll \& Woosley 2013). The He-envelope mass required to trigger the second detonation can drop significantly from $\sim 0.1$ to $\sim 10^{-3} M_{\odot}$ for a WD mass increasing from 0.8 to $1.3 M_{\odot}$ (Fink et al. 2010), while observationally, an $\mathrm{He}$ envelope below $0.05 M_{\odot}$ is favored due to the discrepancy with the theoretical light curve when a massive He envelope is applied (Woosley \& Kasen 2011). 
Such diversities in the He-detonation trigger and detonation wave interactions have provided the flexibility to account for the diversity of SNe Ia. In Diehl et al. (2015), a multiple-plume structure is proposed to illustrate the apparent flickering of the ${ }^{56}$ Co decay line. Based on our models, it is possible that such a feature can be realized by multiple spots in the He envelope. For a quantitative comparison, three-dimensional models are required for a one-to-one matching of the observables and predicted signatures. Nevertheless, using the virtue that geometric convergence and laminar wave shock collision do not differentiate between two- and three-dimensional simulations, our models can shed light on what kind of shock interaction, and hence what kind of detonation pattern, is necessary for reproducing features taken from SN 2014J.

\subsection{How Typical Is SN 2014J?}

In this work, we explore the possible triggering and ignition mechanisms that might lead to the asymmetric properties demonstrated by the observational features of SN 2014J. Among all models, the closest model we obtain is model 110050-R, with a metallicity $\gtrsim 4 Z_{\odot}$.

The total (WD) mass required is $1.0-1.1 M_{\odot}$ at the intermediate-to-high-mass end of a sub-Chandrasekhar-mass WD. Stellar evolution theory suggests a progenitor mass constrained in a range likely to be $\sim 6-7 M_{\odot}$ (see, e.g., Catalán et al. 2008; Doherty et al. 2015 for the progenitor final mass relation) ${ }^{10}$ For a sub-Chandrasekhar-mass WD to produce $0.6 M_{\odot}{ }^{56} \mathrm{Ni}$ as a normal SN Ia, theory suggests an initial mass of $\sim 1.0 M_{\odot}$ in one-dimensional models (Shigeyama et al. 1992; Nomoto \& Leung 2018; Shen et al. 2018). The ${ }^{56} \mathrm{Ni}$ mass production is known to be sensitive to the progenitor mass because of the density dependence of ${ }^{56} \mathrm{Ni}$ production, with a minimum $>5 \times 10^{7} \mathrm{~g} \mathrm{~cm}^{-3}$. Hence, a higher WD mass ensures not only a higher central density but also a higher energy release at the center, which favors the propagation of the detonation wave. In the one-dimensional models, the $1.1 M_{\odot}$ case gives rise to a bright SN Ia for its $\sim 0.8 M_{\odot}$ production of ${ }^{56} \mathrm{Ni}$. However, in multidimensional models, there are variations in the ${ }^{56} \mathrm{Ni}$ production based on the initial He-detonation structure. The aspherical He detonation tends to give a lower ${ }^{56} \mathrm{Ni}$ due to off-center ignition of $\mathrm{C}+\mathrm{O}$ detonation because of the density gradient, as discussed in previous sections.

The He-envelope mass required by our calculations is $\sim 0.05 M_{\odot}$. This is a marginal value for the He detonation to be observed (Woosley \& Kasen 2011), where the optical observational features remain compatible with the data of normal SNe Ia. A high He-envelope mass likely overproduces some iron-peak elements, including $\mathrm{Cr}$ and $\mathrm{V}$, near the surface. This changes the typical isotope and element abundance distributions compared to normal SNe Ia, where such elements are produced in a deeper layer, e.g., the Chandrasekhar-mass WD with a deflagration-detonation transition. Such highopacity material in the envelope may make the explosion appear redder in optical spectra (Polin et al. 2019). However, their results are based on a one-dimensional model where the He-rich matter is always burned from high to low density.

\footnotetext{
${ }^{10}$ However, we remark that at about $7 M_{\odot}$, the final remnant mass is close to the transition mass of the CO WD, where it is possible that the core may have undergone advanced burning that destroyed ${ }^{12} \mathrm{C}$ and produced ${ }^{20} \mathrm{Ne}$, leaving a hybrid $\mathrm{O}+\mathrm{Ne}+\mathrm{Mg}$ core with a $\mathrm{C}+\mathrm{O}$ envelope. The exact transition mass depends on the stellar evolution code and input physics.
}

Therefore, the high-density matter always has a longer time to carry out nuclear reactions, which favors the production of such iron-peak elements. However, for aspherical detonation, this is not always true. The $\mathrm{Cr}$ and $\mathrm{V}$ production depends on how the He detonation spreads around the He envelope.

Another theoretical uncertainty is the exact He mass when the first nuclear runaway starts. The exact He-envelope mass depends on the mass accretion rate and the type of binary system (single- or double-degenerate). A higher $M_{\mathrm{He}}$ is more likely from the double-degenerate scenario, while a lower $M_{\mathrm{He}}$ is more likely from the single-degenerate scenario. Such a calculation was done by Kawai et al. (1988) from the stellar evolutionary perspective. The steady-state accretion of $\mathrm{He}$ on $\mathrm{C}+\mathrm{O}$ and $\mathrm{O}+\mathrm{Ne}+\mathrm{Mg}$ WDs is investigated in the singledegenerate scenario. It is shown that the He-envelope mass drops sharply with the $\mathrm{C}+\mathrm{O}$ core mass, with $\sim 10^{-2} M_{\odot}$ for a $0.7 M_{\odot} \mathrm{C}+\mathrm{O}$ core down to $\sim 10^{-6} M_{\odot}$ for a $1.36 M_{\odot}$ core. This shows that the steady-state accretion in the singledegenerate scenario may not provide a robust way for accumulating an He envelope beyond $10^{-2} M_{\odot}$ in a WD of mass $1.00 M_{\odot}$ or above.

The detonation required at the beginning is from a ring around the equator. Such a configuration is shown to produce more aspherical features in the Ni distribution, which would be compatible with the multiple redshifted ${ }^{56} \mathrm{Co}$ decay lines measured in SN 2014J. But we have also found that other types of initial detonation, such as the D and B types, may also produce similar characteristics, although less pronounced. How the He detonation is initialized is a matter of debate.

For a one-dimensional model, an entire mass shell is ignited simultaneously because of the assumed symmetry. However, it is unclear whether such symmetry can be maintained prior to the ignition. For example, in the single-degenerate scenario, the accretion of matter from the companion star through Roche lobe overflow generally has a high angular momentum. Such rapidly rotating matter, when accumulated on the stellar surface, may create strong dragging, which disturbs the material near the surface. Also, the He burning near the core-envelope interface may trigger convective motion (see, e.g., Jacobs et al. 2016). This creates a highly turbulent background due to the shear between the quasi-static $\mathrm{C}+\mathrm{O}$ core and the rapidly rotating He-rich matter.

Knowing that the runaway of $\mathrm{He}$ is highly temperaturesensitive, it is conceivable that the ignition may occur at random locations and spherical symmetry is broken. In the most extreme case, only one spot can be ignited, which corresponds to the B-type explosion. If the rotation symmetry may be preserved, then an R-type explosion is one of the possibilities. However, the exact configuration will be best estimated from the detailed multidimensional hydrodynamics simulations for the last minutes before the runaway to capture how all of these processes interfere with each other (see, e.g., Zingale et al. 2011; Malone et al. 2014).

The early ${ }^{56} \mathrm{Ni}$ signal can act as a tracer of the explosion mechanism. In Figure 5, we show that how the He detonation is initiated can strongly influence the surface ejecta composition and its angular dependence. In particular, the early low-redshift ${ }^{56} \mathrm{Ni}$ line implies the possibility that we are observing SN 2014J close to where the shock is initialized. If we observe the shock converging point, the abundant elements of ${ }^{28} \mathrm{Si}$ and ${ }^{4} \mathrm{He}$ may easily block the gamma rays. The later ${ }^{56} \mathrm{Co}$ line can have an origin in multiple shock convergence on the He envelope. This 
can be triggered by, for example, multiple rings or bubbles with nonuniform orientation. However, the exact details may require future study because the multiple-plume feature (Diehl et al. 2015) indicates the history of multiple shock locations when the carbon detonation is triggered.

Our explorations favor a high metallicity for the WD compared to the solar metallicity. In fact, this feature is common to the observed $\mathrm{SNe}$ Ia whose chemical abundance is extracted from their light curves and spectra. Such high metallicity appears to be common in recently observed SNe Ia, e.g., SN 2012cg (Graur et al. 2016; Leung \& Nomoto 2018, 2020a) and SNR 3C 397 (Yamaguchi et al. 2015; Leung \& Nomoto 2018). These works demonstrated that a supersolar metallicity is paramount to boost certain isotope or element ratios, especially the ${ }^{57} \mathrm{Ni} /{ }^{56} \mathrm{Ni}$ or $\mathrm{Mn} / \mathrm{Ni}$ ratios. Such effects cannot be completely replaced by tuning other major parameters, such as the WD mass, $M$, or the nuclear runaway structure. These examples demonstrated that the metallicity of exploding WDs can be higher than solar metallicity. A detailed evolutionary path for such a high-metallicity WD progenitor would be interesting future work.

\subsection{Dependence on Model Dimensionality}

We note that there exist controversies regarding twodimensional modeling containing symmetries, which might not necessarily be realized in reality. Ideally, three-dimensional models are required to provide a comprehensive and selfconsistent explanation to match the explanation in a one-to-one correspondence. Here we briefly recapitulate how we use twodimensional models and why this can still provide reliable estimates.

First, two-dimensional models allow a more time-effective search of appropriate models. As indicated in our previous works (Papers I and II), the parameter space that is suitable for $\mathrm{SNe} \mathrm{Ia}$ is large. The running time for one $n$-dimensional hydrodynamical model scales with $N^{n}$ for a grid mesh of $N^{n}$. Typical resolution requires $\sim 500$ grids for one direction. This means that three-dimensional models are, at minimum, $\sim 500$ times more computationally expensive. It generally takes 3-5 days for our two-dimensional model to complete its hydrodynamical simulation and nucleosynthesis. This simple scaling implies that a three-dimensional model requires, at minimum, months for a single model. This is beyond the computational time we can afford for a practical model investigation.

Second, the large-scale aspherical effect can be well captured by two-dimensional models. We recall that two-dimensional models are capable of naturally producing three-dimensional aspherical structure, such as a bubble or ring, as well as onedimensional spherical structure. Three-dimensional simulations can produce more complex structures in the form of multiple bubbles, for example. In fact, the processes determining how the second detonation starts depend on the wave collision details. These can already be captured by one of the twodimensional scenarios. Furthermore, two-dimensional models provide the minimum perturbation from the spherical symmetry. Notice that SN 2014J has features that can be explained by the classical W7 model in a broader picture, even though it has aspherical features. Therefore, it might be considered a generalization to start from models that behave almost spherically and extend them to lower symmetries.

Third, the symmetry is conserved in the simulation. As demonstrated in Moll \& Woosley (2013), where one-, two-, and three-dimensional simulations of the sub-Chandrasekharmass models are carried out, one of their explicit threedimensional models with a two-dimensional counterpart gives results that agree with each other. This provides some support that in the explosion phase, symmetry does not break during its propagation. Similar three-dimensional models in Gronow et al. (2020) also demonstrated similar features, in that the detonation propagates like a two-dimensional front. Furthermore, in this work, we further show that the two-dimensional spherical models explode spherically, as in the one-dimensional case. Both results support that our axisymmetric model remains axisymmetric throughout the simulations, as long as turbulent motion is unimportant. This is true during the explosion phase $(\sim 1 \mathrm{~s})$. When time is sufficiently long, i.e., in the nebular phase, we expect that the Rayleigh-Taylor instabilities play a role and perturb the morphology. Then, initial seeds break the rotation symmetry. However, such effects are secondary compared to the large-scale asymmetry and require more time to grow. Also, similar to models in the literature (see, e.g., Fink et al. 2007, 2010; Sim et al. 2010), we set up WDs in hydrostatic equilibrium as the initial condition. The quiet environment suggests that the turbulent motion is suppressed.

Finally, in this work, we focus on the common features that exist in both two- and three-dimensional models. In particular, we investigate how shock waves superpose, interact with each other, or grow by themselves through geometric convergence and consequently generate the structure that breaks the spherical symmetry. As indicated in Paper II, how the wave interacts is independent of the boundary condition. Thus, our two-dimensional model can offer the necessary starting point to explore which kind of detonation structure is necessary to generate the corresponding interaction for creating the largescale asymmetry.

\subsection{Conclusion}

In this paper, we explored the parameter space in the classical double-detonation model that can produce observables indicating deviations from spherical symmetry. We studied how the initial detonation geometry affects the final explosion morphology by examining the ejecta composition in the spatial distribution and velocity space. We studied how the spherical symmetry can be broken for creating large-scale asymmetry. The sub-Chandrasekhar-mass WD progenitor tends to produce more pronounced asymmetry than the Chandrasekhar-mass WD progenitor. The surface He detonation can be the origin of the early ${ }^{56} \mathrm{Ni}$ gamma-ray line of some SNe Ia, e.g., SN 2014J, and the recently observed early bumps in the observed light curves of some SNe Ia.

We have examined how the initial mass and He-detonation geometry affect the final explosion results, in particular the ejecta geometry and element distribution in both spatial and velocity phase space. We observe that starting the $\mathrm{He}$ detonation as a bubble (with the lowest symmetry), then to a ring, and then a sphere (with the highest symmetry) may give observable differences in the ejecta morphology and velocity for the characteristic elements, including $\mathrm{He}, \mathrm{O}, \mathrm{Si}, \mathrm{S}, \mathrm{Fe}$, and $\mathrm{Ni}$, and their directional dependence.

We have provided a detailed case study on searching for models that may resemble the qualitative features observed in SN 2014J based on the gamma-ray line detections and the latetime photometry of the optical band. Four key aspects of SN Ia explosion are 
(1) the total mass $M$ of the WD determines the total ${ }^{56} \mathrm{Ni}$ production,

(2) the He-envelope mass $M_{\mathrm{He}}$ determines the large-scale asymmetry in the radial distribution of ${ }^{56} \mathrm{Ni}$,

(3) the metallicity determines the required ${ }^{57} \mathrm{Ni} /{ }^{56} \mathrm{Ni}$ mass, and

(4) the initial He-runaway geometry determines the surface ${ }^{56} \mathrm{Ni}$ distribution.

From our explorations simulating a set of key scenarios, we conclude that the SN 2014J progenitor should have the following properties:

(1) an initial He detonation in the orbital plane set by the binary companion,

(2) a WD mass in the range from 1.00 to $1.10 \mathrm{M}_{\odot}$,

(3) a WD metallicity in the range from 3 to $5 Z_{\odot}$, and

(4) an He-envelope mass of $\sim 0.05-0.10 M_{\odot}$.

We also derived the detailed velocity distributions of some major isotopes, for example ${ }^{16} \mathrm{O},{ }^{28} \mathrm{Si},{ }^{54} \mathrm{Fe}$, and ${ }^{56-58} \mathrm{Ni}$, and the spatial distributions of major iron-peaked elements, including $\mathrm{Cr}, \mathrm{Mn}, \mathrm{Fe}$, and $\mathrm{Ni}$. Future observations of the ejecta morphology by specific elements (e.g., Seitenzahl et al. 2019) can provide a strong constraint on the models presented in this work. Large-scale features in these objects might reveal how the detonation has interacted during its propagation, thus shedding light on its initial detonation pattern.

At last, we discuss the recent application of SN tomography as presented in Seitenzahl et al. (2019) for the SNRs 0519-69.0, 0509-67.5, and N103B. From how aspherical the SN ejecta in the reverse-shock-heated region and their corresponding $\mathrm{Fe}$ masses are, we can deduce the fundamental properties of the progenitor, including whether it is a Chandrasekhar or subChandrasekhar WD, the expected initial mass, and the detonation geometry. We also summarize the $M$ and $Z$ dependence of the major element ratios typically found in the spectra of SNRs.

This work has been supported by the World Premier International Research Center Initiative (WPI Initiative), MEXT, Japan. S.C.L. acknowledges support from grants HST-AR-15021.001-A and 80NSSC18K1017. K.N. acknowledges support from JSPS KAKENHI grant Nos. JP17K05382 and JP20K04024. T.S. is supported by the German Research Society (DFG-Forschungsstipendium SI 2502/1-1). This research was also supported by the DFG cluster of excellence "Origin and Structure of the Universe." This article is also supported through the "ChETEC" COST Action (CA16117).

\section{Appendix WD Models for SNe Ia}

The SNe Ia are the thermonuclear explosions of CO WDs (see, e.g., Hillebrandt \& Niemeyer 2000; Nomoto \& Leung 2017). A single CO WD does not spontaneously undergo nuclear burning. In a close binary system, on the other hand, the WD gains mass by mass transfer from its companion star, which includes a slightly evolved near-main-sequence star, a red giant, and an He star (single-degenerate scenario; e.g., Nomoto 1982a; Kawai et al. 1988) or a WD (double-degenerate scenario; Iben \& Tutukov 1984; Webbink 1984).

During the mass accretion, if the accretion rate is relatively low, the accreted $\mathrm{He}$ is accumulated on the surface, and eventually, He burning is ignited, first in the off-center hot spot when the WD mass is the sub-Chandrasekhar mass
(Nomoto 1982a). Then, He detonation is developed (Nomoto 1982b).

If the accretion rate is relatively high, on the other hand, the WD mass reaches the near-Chandrasekhar mass, and the central temperature exceeds $\sim 3 \times 10^{8} \mathrm{~K}$. Then, the energy-generation rate of ${ }^{12} \mathrm{C}$ burning exceeds the neutrino cooling rate. The ${ }^{12} \mathrm{C}$ burning is unable to develop a $\mathrm{C}$ deflagration in the center (Nomoto 1982a). ${ }^{11}$

\section{A.1. Near-Chandrasekhar-mass WD Models}

When ${ }^{12} \mathrm{C}$ burning is ignited in the center of the nearChandrasekhar-mass WD, the central density is as high as $2-3 \times 10^{9} \mathrm{~g} \mathrm{~cm}^{-3}$. Electrons are strongly degenerate, so the gas pressure is not so sensitive to the temperature. The temperature rise becomes unregulated, as the carbon-burning rate is strongly temperature-sensitive $\left(\sim T^{33}\right)$. This plants the first seed of nuclear runaway. Simultaneously, the rapid temperature rise does not trigger a shock because the pressure growth is small compared to the temperature rise due to the strong degeneracy, even when the central temperature becomes as high as $\sim 10^{10} \mathrm{~K}$ (Nomoto et al. 1976; Nomoto \& Sugimoto 1977). As a result, the temperature jump becomes a localized event, where the temperature discontinuity propagates by microscopic processes instead of macroscopic shock compression. Thus, the initial runaway is likely to be in the form of a deflagration wave (Nomoto et al. 1976, 1984). The short mean free path of electron conduction in such a density implies a very thin flame front $\left(\sim 10^{-3} \mathrm{~cm}\right)$ compared to the size of a WD $\left(\sim 10^{3} \mathrm{~km}\right.$; Timmes \& Woosley 1992).

Despite the turbulent motion emerging down to the Kolmogorov scale $\left(10^{-3} \mathrm{~cm}\right.$, assuming a typical Reynolds number of $\sim 10^{14}$ ), the Gibson scale decreases with density and is generally larger than the Kolmogorov scale. Flame structure with a size below the Gibson scale $\left(\sim 10^{-1} \mathrm{~km}\right.$ at the center) is smoothed (Niemeyer \& Hillebrandt 1995; Röpke et al. 2003, 2004a), albeit eddies can appear below this scale.

If the propagation speed of the subsonic deflagration is fast enough, the deflagration efficiently releases the necessary energy for unbinding a WD and creates a successful explosion like the W7 model (Nomoto et al. 1984), as does the detonation wave (Arnett 1969).

The explosive nuclear burning at high densities synthesizes iron-peak elements (Thielemann et al. 1986; Iwamoto et al. 1999). However, the observed intermediate-mass elements should be synthesized at lower densities, which suggests that the explosion consists of subsonic burning, i.e., deflagration (Nomoto et al. 1976; Nomoto \& Sugimoto 1977), which decreases the densities at the flame front.

If the propagation of the deflagration wave is slow, it may not unbind the star (but in some cases may cause pulsation) because the stellar expansion makes the deflagration wave quench (Nomoto et al. 1976). A subsequent transition from deflagration to detonation is vital for explaining a successful SN Ia explosion, known as the deflagration-detonation transition (Nomoto et al. 1984; Khokhlov 1991; Arnett \& Livne 1994; Iwamoto et al. 1999; Gamezo et al. 2003, 2004; Röpke et al. 2007). However, the deflagration-detonation transition requires a turbulence strength that is less likely to be

\footnotetext{
11 Depending on the mass accretion rate, the WD can undergo centered deflagration, double detonation, or accretion-induced collapse (see also Nomoto 1982b; Nomoto et al. 1984, for a detailed phase diagram).
} 
reached (see, e.g., Khokhlov et al. 1997; Niemeyer et al. 1999; Lisewski et al. 2000; Gamezo et al. 2005; Woosley et al. 2009). In order to realize this effect, ab initio numerical experiments with very fine resolutions $(\sim 0.1 \mathrm{~km})$ are necessary (Kushnir et al. 2012) that are 1-2 orders of magnitude below the affordable resolution. Recent direct experiments, both numerical and laboratory ones using a methane-air mixture to explicitly resolve turbulent motion, have demonstrated that turbulent acceleration can be an important key factor (Poludenko et al. 2011; Poludnenko et al. 2019).

The motion of the deflagration wave can be convoluted. The subsonic propagation ( $\sim 1 \%$ of the speed of sound) implies that the deflagration wave structure is coupled with the underlying fluid motion, which means that the flame structure is also susceptible to various hydrodynamics instabilities, such as Rayleigh-Taylor (Bell et al. 2004a; Zingale et al. 2005; Hicks 2015, 2019), Kelvin-Helmholtz, Landau-Derrieus (Bell et al. 2004b; Röpke et al. 2004b), and pulsational (Glazyrin 2013, 2014; Glazyrin et al. 2013; Poludnenko 2015) instabilities. On the contrary, the supersonic detonation is less sensitive to fluid motion. However, direct numerical simulations of the small-scale detonation show spontaneous cellular structure formation behind the detonation wave front (Gamezo et al. 1999).

To model the explosion, following how the deflagration propagates reveals how the energy is released. The subgrid scale of the reaction front indicates that on-site modeling is inaccurate, but a subgrid-scale model is necessary to describe partial cell burning and the irregular wave front inside the cell. This relies on the subgrid-scale turbulence model (Clement 1993; Niemeyer \& Hillebrandt 1995; Shih et al. 1995a, 1995b) and the flame-tracking scheme.

The subgrid-scale turbulence model assumes that eddy motion below the resolved scale can be well described by statistical models. This gives an accurate approximation given the large difference between the resolved scale and the much smaller Kolmogorov scale. Scaling relation has been studied explicitly in direct simulations (see, e.g., Fisher et al. 2019). The model tracks the generation and dissipation of eddy motions by channels including shear stress, compression, Rayleigh-Taylor instabilities, and so on (see, e.g., Niemeyer \& Hillebrandt 1995; Shih et al. 1995a, 1995b; Schmidt et al. 2006).

Flame-tracking schemes are algorithms designed for resolving subgrid-scale features. There are multiple representations, including (1) the advective-diffusive-reactive equations (Khokhlov 1995; Vladimirova et al. 2006; Townsley et al. 2007), (2) level-set methods (Osher \& Sethian 1988; Sethian 2001), and (3) point-set methods (Glimm et al. 1999, 2000; Zhang 2009; Leung et al. 2015a). The main idea is to introduce additional variables with model parameters that represent how much the grid is partially burned; from that, the actual flamefront geometry is reconstructed.

\section{A.2. Sub-Chandrasekhar-mass WD Models}

In a sub-Chandrasekhar-mass WD, the less degenerate matter with a lower density means that the detonation is more likely (Nomoto 1982a). Such a scenario is viable when the surface energy production is faster than its heat loss by convection or expansion (Jacobs et al. 2016). The initial nuclear runaway can be triggered by accretion from its companion star in the single-degenerate scenario or through a violent merger in the double-degenerate scenario (see, e.g., Tanikawa et al. 2015, 2019). Pure CO matter has a high ignition threshold for its high temperature $\left(\sim 1-2 \times 10^{9} \mathrm{~K}\right.$; e.g., Sato et al. 2015), which is shown to be difficult to trigger and sensitive to the way of contact (Dan et al. 2012). A WD merger with a helium envelope can suppress this ignition condition, but its required hot-spot size can be unrealizable in a thin WD envelope (see, e.g., Shen \& Bildsten 2009). Mixing with $\mathrm{C} / \mathrm{O}$-rich matter through, for example, turbulence can resolve this difficulty (Holcomb et al. 2013; Piro 2015). The violent merger of two CO WDs (Pakmor et al. 2012) is therefore challenging for a robust ignition, as the collision can fail to generate spots that are sufficiently hot for the first runaway (Dan et al. 2012, 2014). Mixture with helium provided by its He envelope or the companion star lowers the ignition temperature such that the detonation trigger is less sensitive to the merging dynamics (Shen \& Moore 2014). A thin layer of $\mathrm{He}\left(\sim 0.01 M_{\odot}\right)$ can already trigger the second explosion more robustly (Pakmor et al. 2013). When the He detonation fails to trigger the second detonation, the star develops like a nova and explodes as a so-called SN Ia (Bildsten et al. 2007; Shen et al. 2010; Waldman et al. 2011).

Even when the $\mathrm{C}$-detonation trigger becomes robust with the aid of $\mathrm{He}$, the exact position and timing of the detonation are unclear because they depend on the dynamics of the $\mathrm{He}$ atmosphere. These require multidimensional low Mach number simulations of the atmosphere for multiple eddy turnover times. Multiple possibilities exist, including direct $\mathrm{He}$ ignition, $\mathrm{C}$ ignition, or ignition after the merger process when a Chandrasekhar-mass WD is formed (Dan et al. 2011; Shen et al. 2018; Tanikawa et al. 2019). Geometric convergence in a low-mass WD is more difficult to achieve (Shen \& Moore 2014). High-resolution simulations using the ab initio approach are necessary to trace when and where the first hot spot appears (Fenn \& Plewa 2017). The asymmetry in a threedimensional simulation tends to suppress the prompt detonation as the geometrical convergence breaks down in the violent merger scenario (Fenn et al. 2016).

In a low-mass WD, the detonation front has a size comparable with the resolved scale. This allows direct coupling of the hydrodynamics with a nuclear reaction network (Shen et al. 2018; Polin et al. 2019). In a more massive CO WD (central density $\geqslant 10^{8} \mathrm{~g} \mathrm{~cm}^{-3}$ ), the detonation width can be much smaller than the resolved grid size $(\sim 10 \mathrm{~km})$. Subgridscale methods or adaptive mesh refinement are often used in the literature.

\section{ORCID iDs}

Shing-Chi Leung (iD https://orcid.org/0000-0002-4972-3803 Roland Diehl (1) https://orcid.org/0000-0002-8337-9022 Ken'ichi Nomoto (iD https://orcid.org/0000-0001-9553-0685 Thomas Siegert (i) https://orcid.org/0000-0002-0552-3535

\section{References}

Arnett, D., \& Livne, E. 1994, ApJ, 427, 314

Arnett, W. D. 1969, Ap\&SS, 5, 180

Ashall, C., Mazzali, P., Bersier, D., et al. 2014, MNRAS, 445, 4427

Badenes, C., Borkowski, K. J., \& Bravo, E. 2005, ApJ, 624, 198

Badenes, C., Borkowski, K. J., Hughes, J. P., Hwang, U., \& Bravo, E. 2006, ApJ, 645, 1373

Badenes, C., Bravo, E., \& Hughes, J. P. 2008, ApJL, 680, L33

Bell, J. B., Day, M. S., Rendleman, C. A., Woosley, S. E., \& Zingale, M. 2004a, ApJ, 608, 883 
Bell, J. B., Day, M. S., Rendleman, C. A., Woosley, S. E., \& Zingale, M. 2004b, ApJ, 606, 1029

Benvenuto, O. G., Panei, J. A., Nomoto, K., Kitamura, H., \& Hachisu, I. 2015, ApJL, 809, L6

Bildsten, L., Shen, K. J., Weinberg, N. N., \& Nelemans, G. 2007, ApJL, 662, L95

Blondin, S., Dessart, L., \& Hillier, D. J. 2018, MNRAS, 474, 3931

Brown, P. J., Smitka, M. T., Wang, L., et al. 2015, ApJ, 805, 74

Bulla, M., Sim, S. A., Kromer, M., et al. 2016, MNRAS, 462, 1039

Catalán, S., Isern, J., García-Berro, E., \& Ribas, I. 2008, MNRAS, 387, 1693

Churazov, E., Sunyaev, R., Isern, J., et al. 2014, Natur, 512, 406

Churazov, E., Sunyaev, R., Isern, J., et al. 2015, ApJ, 812, 62

Clement, M. J. 1993, ApJ, 406, 651

Dan, M., Rosswog, S., Brüggen, M., \& Podsiadlowski, P. 2014, MNRAS, 438, 14

Dan, M., Rosswog, S., Guillochon, J., \& Ramirez-Ruiz, E. 2011, ApJ, 737, 89

Dan, M., Rosswog, S., Guillochon, J., \& Ramirez-Ruiz, E. 2012, MNRAS, 422, 2417

Dave, P., Kashyap, R., Fisher, R., et al. 2017, ApJ, 841, 58

de los Reyes, M. A. C., Kirby, E. N., Seitenzahl, I. R., \& Shen, K. J. 2020, ApJ, 891,85

Dhawan, S., Flörs, A., Leibundgut, B., et al. 2018, A\&A, 619, A102

Diehl, R. 2015, AN, 336, 464

Diehl, R., Siegert, T., Hillebrandt, W., et al. 2014, Sci, 345, 1162

Diehl, R., Siegert, T., Hillebrandt, W., et al. 2015, A\&A, 574, A72

Doherty, C. L., Gil-Pons, P., Siess, L., Lattanzio, J. C., \& Lau, H. H. B. 2015 , MNRAS, 446, 2599

Dragulin, P., \& Hoeflich, P. 2016, ApJ, 818, 26

Fenn, D., \& Plewa, T. 2017, MNRAS, 468, 1361

Fenn, D., Plewa, T., \& Gawryszczak, A. 2016, MNRAS, 462, 2486

Ferrand, G., Warren, D. C., Ono, M., et al. 2019, ApJ, 877, 136

Fink, M., Hillebrandt, W., \& Röpke, F. K. 2007, A\&A, 476, 1133

Fink, M., Röpke, F. K., Hillebrandt, W., et al. 2010, A\&A, 514, A53

Fink, M., Kromer, M., Seitenzahl, I. R., et al. 2014, MNRAS, 438, 1762

Fisher, R., Mozumdar, P., \& Casabona, G. 2019, ApJ, 876, 64

Foley, R. J., Fox, O. D., McCully, C., et al. 2014, MNRAS, 443, 2887

Fry, B. J., Fields, B. D., \& Ellis, J. R. 2015, ApJ, 800, 71

Gamezo, V. N., Khokhlov, A. M., \& Oran, E. S. 2004, PhRvL, 92, 211102

Gamezo, V. N., Khokhlov, A. M., \& Oran, E. S. 2005, ApJ, 623, 337

Gamezo, V. N., Khokhlov, A. M., Oran, E. S., Chtchelkanova, A. Y., \& Rosenberg, R. O. 2003, Sci, 299, 77

Gamezo, V. N., Wheeler, J. C., Khokhlov, A. M., \& Oran, E. S. 1999, ApJ, 512,827

García-Senz, D., Cabezón, R. M., \& Domínguez, I. 2018, ApJ, 862, 27

Glasner, S. A., Livne, E., Steinberg, E., Yalinewich, A., \& Truran, J. W. 2018, MNRAS, 476, 2238

Glazyrin, S. I. 2013, AstL, 39, 221

Glazyrin, S. I. 2014, Ap\&SS, 350, 683

Glazyrin, S. I., Blinnikov, S. I., \& Dolgov, A. 2013, MNRAS, 433, 2840

Glimm, J., Grove, J. W., Li, X., \& Zhao, N. 1999, Contemp. Math., 238, 133

Glimm, J., Grove, J. W., Li, X. L., \& Tan, D. C. 2000, SIAM Journal on Scientific Computing, 21, 2240

Goobar, A., Johansson, J., Amanullah, R., et al. 2014, ApJL, 784, L12

Graur, O., \& Woods, T. E. 2019, MNRAS, 484, L79

Graur, O., Zurek, D., Shara, M. M., et al. 2016, ApJ, 819, 31

Grefenstette, B. W., Fryer, C. L., Harrison, F. A., et al. 2017, ApJ, 834, 19

Grefenstette, B. W., Harrison, F. A., Boggs, S. E., et al. 2014, Natur, 506, 339

Gronow, S., Collins, C., Ohlmann, S. T., et al. 2020, A\&A, 635, A169

Hicks, E. P. 2015, ApJ, 803, 72

Hicks, E. P. 2019, MNRAS, 489, 36

Hillebrandt, W., \& Niemeyer, J. C. 2000, ARA\&A, 38, 191

Holcomb, C., Guillochon, J., de Colle, F., \& Ramirez-Ruiz, E. 2013, ApJ, 771,14

Hughes, J. P., Hayashi, I., Helfand, D., et al. 1995, ApJL, 444, L81

Iben, I. J., \& Tutukov, A. V. 1984, ApJS, 54, 335

Isern, J., Jean, P., Bravo, E., et al. 2016, A\&A, 588, A67

Iwamoto, K., Brachwitz, F., Nomoto, K., et al. 1999, ApJS, 125, 439

Jack, D., Mittag, M., Schröder, K. P., et al. 2015, MNRAS, 451, 4104

Jacobs, A. M., Zingale, M., Nonaka, A., Almgren, A. S., \& Bell, J. B. 2016, ApJ, 827, 84

Jiang, J.-A., Doi, M., Maeda, K., et al. 2017, Natur, 550, 80

Jiang, J.-A., Doi, M., Maeda, K., \& Shigeyama, T. 2018, ApJ, 865, 149

Kawabata, K. S., Akitaya, H., Yamanaka, M., et al. 2014, ApJL, 795, L4

Kawai, Y., Saio, H., \& Nomoto, K. 1988, ApJ, 328, 207

Kelly, P. L., Fox, O. D., Filippenko, A. V., et al. 2014, ApJ, 790, 3

Khokhlov, A., Oran, E. S., \& Wheeler, J. C. 1997, ApJ, 478, 678
Khokhlov, A. M. 1991, A\&A, 245, 114

Khokhlov, A. M. 1995, ApJ, 449, 695

Kippenhahn, R., \& Thomas, H. C. 1978, A\&A, 63, 265

Kobayashi, C., Leung, S.-C., \& Nomoto, K. 2020, ApJ, 895, 138

Kushnir, D., Livne, E., \& Waxman, E. 2012, ApJ, 752, 89

Leung, S. C., Chu, M. C., \& Lin, L. M. 2015a, MNRAS, 454, 1238

Leung, S. C., Chu, M. C., \& Lin, L. M. 2015b, ApJ, 812, 110

Leung, S.-C., \& Nomoto, K. 2017a, in Proc. 14th Int. Symp. on Nuclei in the Cosmos (NIC2016), ed. S. Kubono et al. (Tokyo: JPS), 020506

Leung, S. C., \& Nomoto, K. 2017b, MmSAI, 88, 266

Leung, S.-C., \& Nomoto, K. 2018, ApJ, 861, 143

Leung, S.-C., \& Nomoto, K. 2019, PASA, 36, e006

Leung, S.-C., \& Nomoto, K. 2020a, ApJ, 888, 80

Leung, S.-C., \& Nomoto, K. 2020b, ApJ, 900, 54

Leung, S.-C., Nomoto, K., \& Suzuki, T. 2020, ApJ, 889, 34

Leung, S.-C., Zha, S., Chu, M.-C., Lin, L.-M., \& Nomoto, K. 2019, ApJ, 884,9

Lisewski, A. M., Hillebrandt, W., \& Woosley, S. E. 2000, ApJ, 538, 831

Lopez, L. A., Grefenstette, B. W., Reynolds, S. P., et al. 2015, ApJ, 814, 132

Maggi, P., Filipović, M. D., Vukotić, B., et al. 2019, A\&A, 631, A127

Maggi, P., Haberl, F., Kavanagh, P. J., et al. 2016, A\&A, 585, A162

Malone, C. M., Nonaka, A., Woosley, S. E., et al. 2014, ApJ, 782, 11

Maoz, D., Mannucci, F., \& Nelemans, G. 2014, ARA\&A, 52, 107

Margutti, R., Parrent, J., Kamble, A., et al. 2014, ApJ, 790, 52

Martínez-Rodríguez, H., Badenes, C., Lee, S.-H., et al. 2018, ApJ, 865, 151

Martínez-Rodríguez, H., Badenes, C., Yamaguchi, H., et al. 2017, ApJ, 843, 35

McWilliam, A., Piro, A. L., Badenes, C., \& Bravo, E. 2018, ApJ, 857, 97

Moll, R., \& Woosley, S. E. 2013, ApJ, 774, 137

Mori, K., Famiano, M. A., Kajino, T., et al. 2018, ApJ, 863, 176

Nagao, T., Maeda, K., \& Yamanaka, M. 2017, ApJ, 835, 143

Niemeyer, J. C., Bushe, W. K., \& Ruetsch, G. R. 1999, ApJ, 524, 290

Niemeyer, J. C., \& Hillebrandt, W. 1995, ApJ, 452, 769

Nomoto, K. 1982a, ApJ, 253, 798

Nomoto, K. 1982b, ApJ, 257, 780

Nomoto, K., \& Leung, S. 2017, in Handbook of Supernovae, ed. A. W. Alsabti \& P. Murdin (Berlin: Springer)

Nomoto, K., \& Leung, S.-C. 2018, SSRv, 214, 67

Nomoto, K., \& Sugimoto, D. 1977, PASJ, 29, 765

Nomoto, K., Sugimoto, D., \& Neo, S. 1976, Ap\&SS, 39, 37

Nomoto, K., Thielemann, F. K., \& Yokoi, K. 1984, ApJ, 286, 644

Nugent, P. E., Sullivan, M., Cenko, S. B., et al. 2011, Natur, 480, 344

Osher, S., \& Sethian, J. A. 1988, JCoPh, 79, 12

Pakmor, R., Kromer, M., Taubenberger, S., et al. 2012, ApJL, 747, L10

Pakmor, R., Kromer, M., Taubenberger, S., \& Springel, V. 2013, ApJL, 770, L8

Pérez-Torres, M. A., Lundqvist, P., Beswick, R. J., et al. 2014, ApJ, 792, 38 Piro, A. L. 2015, ApJ, 801, 137

Piro, A. L., \& Bildsten, L. 2004, ApJ, 610, 977

Polin, A., Nugent, P., \& Kasen, D. 2019, ApJ, 873, 84

Poludenko, A. Y., Gardiner, T. Y., \& Oran, E. S. 2011, PhRvL, 107, 054501

Poludnenko, A. Y. 2015, PhFl, 27, 014106

Poludnenko, A. Y., Chambers, J., Ahmed, K., Gamezo, V. N., \& Taylor, B. D. 2019, Sci, 366, aau7365

Röpke, F. K., Hillebrandt, W., Schmidt, W., et al. 2007, ApJ, 668, 1132

Röpke, F. K., Hillebrandt, W., \& Niemeyer, J. C. 2004a, A\&A, 420, 411

Röpke, F. K., Hillebrandt, W., \& Niemeyer, J. C. 2004b, A\&A, 421, 783

Röpke, F. K., Niemeyer, J. C., \& Hillebrandt, W. 2003, ApJ, 588, 952

Sato, Y., Nakasato, N., Tanikawa, A., et al. 2015, ApJ, 807, 105

Schmidt, W., Niemeyer, J. C., Hillebrndt, W., \& Roepke, F. K. 2006, A\&A, 450,283

Seitenzahl, I. R., Ghavamian, P., Laming, J. M., \& Vogt, F. P. A. 2019, PhRvL, 123, 041101

Seitenzahl, I. R., Taubenberger, S., \& Sim, S. A. 2009, MNRAS, 400, 531

Sethian, J. 2001, JCoPh, 169, 503

Shen, K. J., \& Bildsten, L. 2009, ApJ, 699, 1365

Shen, K. J., Kasen, D., Miles, B. J., \& Townsley, D. M. 2018, ApJ, 854, 52

Shen, K. J., Kasen, D., Weinberg, N. N., Bildsten, L., \& Scannapieco, E. 2010, ApJ, 715, 767

Shen, K. J., \& Moore, K. 2014, ApJ, 797, 46

Shigeyama, T., Nomoto, K., Yamaoka, H., \& Thielemann, F.-K. 1992, ApJL, 386, L13

Shih, T.-H., Liou, W. W., Shabbir, A., Yang, Z., \& Zhu, J. 1995a, CF, 24, 227

Shih, T.-H., Zhu, J., \& Lumley, J. L. 1995b, CMAME, 125, 287

Siegert, T., \& Diehl, R. 2015, arXiv: 1501.05648

Sim, S. A., Röpke, F. K., Hillebrandt, W., et al. 2010, ApJL, 714, L52

Tanikawa, A., Nakasato, N., Sato, Y., et al. 2015, ApJ, 807, 40 
Tanikawa, A., Nomoto, K., Nakasato, N., \& Maeda, K. 2019, ApJ, 885, 103

Taubenberger, S. 2017, in Handbook of Supernovae, ed. A. W. Alsabti \& P. Murdin (Berlin: Springer), 317

Terada, Y., Maeda, K., Fukazawa, Y., et al. 2016, ApJ, 823, 43

The, L.-S., \& Burrows, A. 2014, ApJ, 786, 141

Thielemann, F. K., Nomoto, K., \& Yokoi, K. 1986, A\&A, 158, 17

Timmes, F. X. 1999, ApJS, 124, 241

Timmes, F. X., Hoffman, R. D., \& Woosley, S. E. 2000, ApJS, 129, 377

Timmes, F. X., \& Woosley, S. E. 1992, ApJ, 396, 649

Townsley, D. M., Calder, A. C., Asida, S. M., et al. 2007, ApJ, 668, 1118

Townsley, D. M., Miles, B. J., Timmes, F. X., Calder, A. C., \& Brown, E. F. 2016, ApJS, 225, 3

Travaglio, C., Hillebrandt, W., Reinecke, M., \& Thielemann, F. K. 2004, A\&A, 425, 1029

Vladimirova, N., Weirs, V. G., \& Ryzhik, L. 2006, CTM, 10, 727

Waldman, R., Sauer, D., Livne, E., et al. 2011, ApJ, 738, 21

Wang, W., \& Li, Z. 2014, ApJ, 789, 123
Wang, X., Fields, B. D., \& Lien, A. Y. 2019, MNRAS, 486, 2910

Webbink, R. F. 1984, ApJ, 277, 355

Wilk, K. D., Hillier, D. J., \& Dessart, L. 2018, MNRAS, 474, 3187

Woosley, S. E., \& Kasen, D. 2011, ApJ, 734, 38

Woosley, S. E., Kerstein, A. R., Sankaran, V., Aspden, A. J., \& Röpke, F. K. 2009, ApJ, 704, 255

Yamaguchi, H., Badenes, C., Foster, A. R., et al. 2015, ApJL, 801, L31

Yamaoka, H., Nomoto, K., Shigeyama, T., \& Thielemann, F.-K. 1992, ApJL, 393, L55

Yang, Y., Wang, L., Baade, D., et al. 2018, ApJ, 852, 89

Zha, S., Chu, M.-C., Leung, S.-C., \& Lin, L.-M. 2019a, ApJ, 883, 13

Zha, S., Leung, S.-C., Suzuki, T., \& Nomoto, K. 2019b, ApJ, 886, 22

Zhang, Y. 2009, Nonli, 22, 1909

Zhou, P., Leung, S.-C., Li, Z., et al. 2021, ApJ, 908, 31

Zingale, M., Nonaka, A., Almgren, A. S., et al. 2011, ApJ, 740, 8

Zingale, M., Nonaka, A., Almgren, A. S., et al. 2013, ApJ, 764, 97

Zingale, M., Woosley, S. E., Rendleman, C. A., Day, M. S., \& Bell, J. B. 2005, ApJ, 632, 1021 